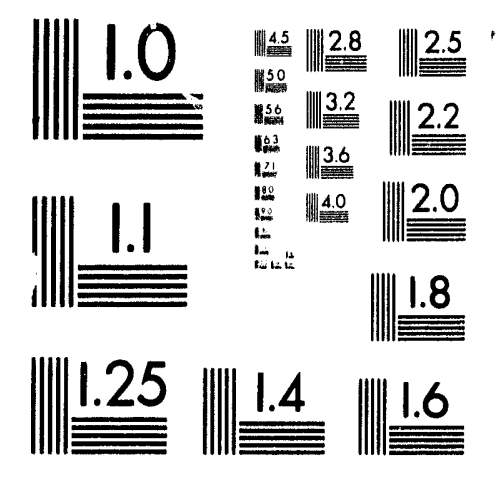



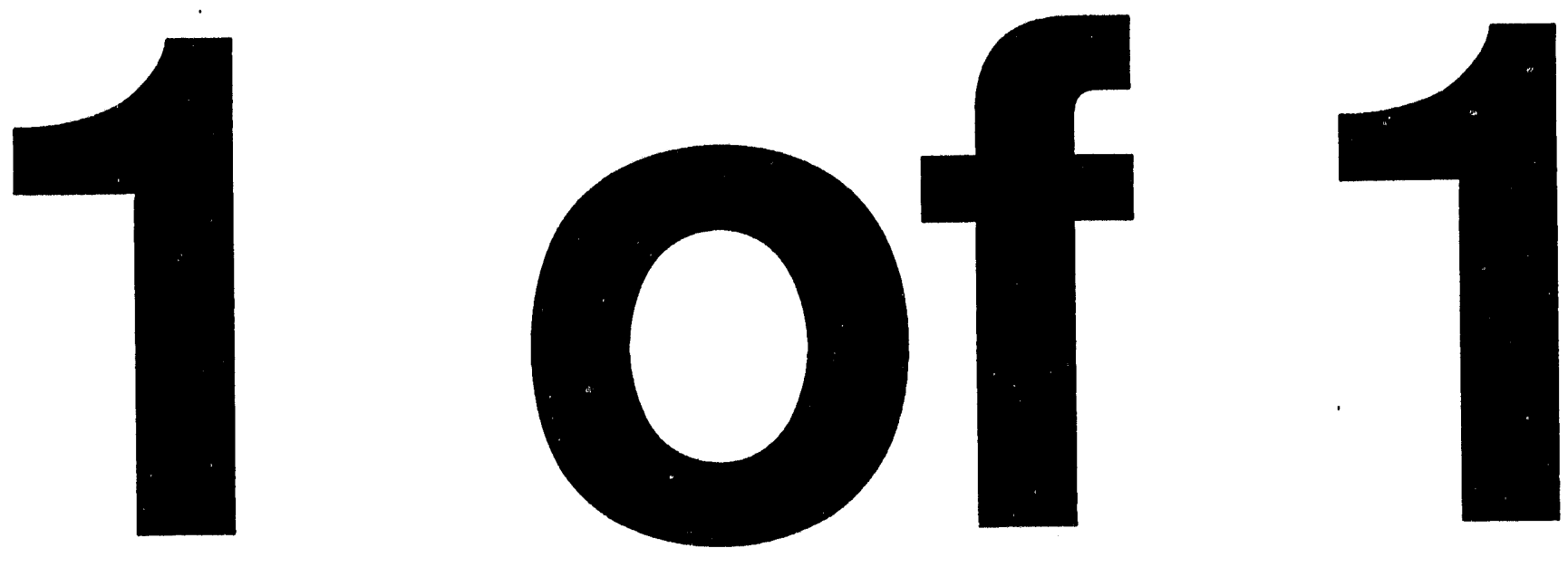
NUREG/CR-6071

ORNL/TM-12406

R5

\section{Impact of ENDF/B-VI \\ Cross-Section Data on \\ H. B. Robinson Cycle 9 \\ Dosimetry Calculations}

Manuscript ('ompleted: September 1993

Date Published: October 1993

Prepared by

M. L. Williams*, M. Asgari*, F. B. K. Kam

Oak Ridge National Laboratory

Operated by Martin Marietta Energy Systems, Inc.

Oak Ridge National Laboratory

Oak Ridge. TN 37831-6285

\section{DISCLAIMER}

This report was prepared as an account of work sponsored by an agency of the United States Government. Neither the United States Government nor any agency thereof, nor any of their employees, makes any warranty, express or implied, or assumes any legal liability or responsibility for the accuracy, completeness, or usefulness of any information, apparatus, product, or process disclosed, or represents that its use would not infringe privately owned rights. Reference herein to any specific commercial product, process, or service by trade name, trademark, manufacturer, or otherwise does not necessarily constitute or imply its endorsement, recommendation, or favoring by the United States Government or any agency thereof. The views and opinions of authors expressed herein do not necessarily state or reflect those of the United States Government or any agency thereof.

Prepared for

Division of Engineering

Office of Nuclear Regulatory Research U.S. Nuclear Regulatory Commission

Washington, DC 20555-0001

NRC FIN B0415

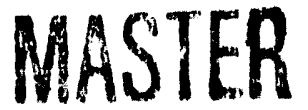

DHSTHBUTION OF THS DOOUMENT IS UNLIMITED

*Louisiana State University Nuclear Science Center Baton Rouge, LA 7()8()3 


\begin{abstract}
Dosimeters that were removed from the H. B. Robinson reactor following Cycle 9 were analyzed and compared with calculated results in an earlier study. This work updates the calculation using recently available ENDF/B-VI data in order to assess advantages to using the newer cross sections in reactor pressure vessel fluence calculations. A comparison is also made to determine the impact of various cross-section libraries on computed dosimeter activities.
\end{abstract}

Significant improvements are obtained with the ENDF/B-VI cross sections. Other factors, such as differences in group structures of multigroup libraries, may also affect the calculated dosimeter activities. 


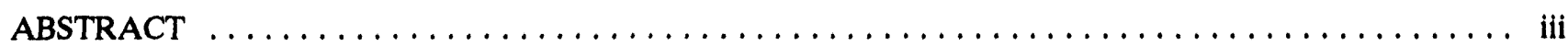

ACKNOWLEDGMENTS $\ldots \ldots \ldots \ldots \ldots \ldots \ldots \ldots \ldots \ldots \ldots \ldots \ldots \ldots \ldots \ldots \ldots \ldots$ viii

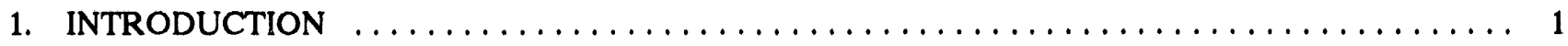

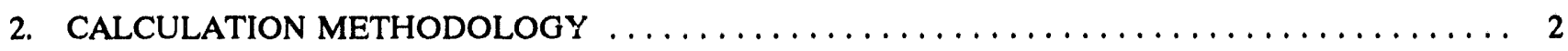

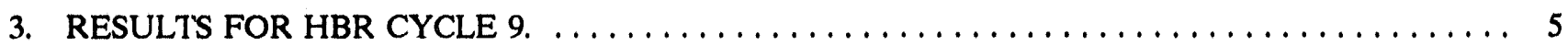

4. COMPARISON OF SAILOR, ELXSIR, AND VITAMIN-E CALCULATIONS FOR A 1-D MODEL OF HBR $\ldots \ldots \ldots \ldots \ldots \ldots \ldots \ldots \ldots \ldots \ldots \ldots \ldots \ldots \ldots \ldots \ldots \ldots$

5. SUMMARY AND CONCLUSIONS $\ldots \ldots \ldots \ldots \ldots \ldots \ldots \ldots \ldots \ldots \ldots \ldots \ldots \ldots \ldots \ldots$

REFERENCES $\ldots \ldots \ldots \ldots \ldots \ldots \ldots \ldots \ldots \ldots \ldots \ldots \ldots \ldots \ldots \ldots \ldots \ldots \ldots \ldots \ldots$ 


\section{LIST OF FIGURES}

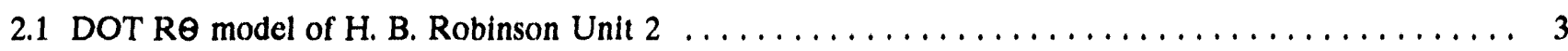

2.2 Ratio of REV-V and ENDF/B-VI inelastic multigroup cross sections to the ENDF/B-V values (natural iron) $\ldots \ldots \ldots \ldots \ldots \ldots \ldots \ldots \ldots \ldots \ldots$ 


\section{LIST OF TABLES}

3.1 Absolute spectra at $20^{\circ}$ surveillance location in HBR Cyclc 9 (calculated with ENDF/B-VI Fe-O-H) .. 6

3.2 Absolute spectra at $0^{\circ}$ cavity location in HBR Cycle 9 (calculated with ENDF/B-VI Fe-O-H) $\ldots \ldots .7$

3.3 Absolute dosimeter response rates for mid-cycle 9 of HBR (calculated with ENDF/B-VI Fe-O-H and Version Vl dosimeter cross sections $) \ldots \ldots \ldots \ldots \ldots \ldots \ldots \ldots \ldots \ldots \ldots \ldots \ldots \ldots$

3.4 Ratio of dosimeter reaction rates obtained with original SAILOR Library to values obtained with ELXSIR $\ldots \ldots \ldots \ldots \ldots \ldots \ldots \ldots \ldots \ldots \ldots \ldots \ldots \ldots \ldots$

3.5 Ratio of dosimeter reaction rates obtained with SAILOR (ENDF/B-VI Fe-O-H) to values obtained with ELXSIR $\ldots \ldots \ldots \ldots \ldots \ldots \ldots \ldots \ldots \ldots \ldots \ldots \ldots \ldots \ldots \ldots$

3.6 Impact of ENDF/B-VI Fe-O-H data on calculated cavity reaction rates for HBR . . . . . . . . . . 9

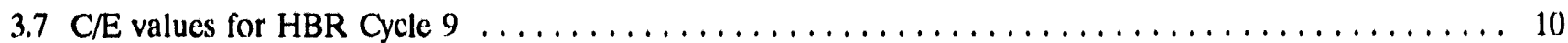

4.1 Impact of self-shielding on fine-group VITAMIN- $\varepsilon_{i}$ calculations in 1-D HBR model $\ldots \ldots \ldots \ldots$

4.2 Impact of different cross-section weighting on ELXSIR calculations in 1-D HBR model $\ldots \ldots \ldots \ldots 13$

4.3 Comparison of SAILOR and ELXSIR calculations for cavity integral fluxes in 1-D HBR model . . . 14

4.4 Comparison of SAILOR and ELXSIR calculations for cavity ${ }^{227} \mathrm{~Np}(\mathrm{n}, \mathrm{f})$ in $1-\mathrm{D}$ HBR model $\ldots \ldots \ldots 14$

4.5 VITAMIN-E, ELXSIR, and SAILOR group structures $\ldots \ldots \ldots \ldots \ldots \ldots \ldots \ldots \ldots \ldots \ldots$

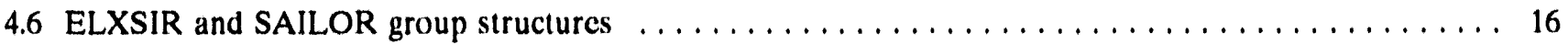




\section{ACKNOWLEDGMENIS}

The authors acknowledge the technical and financial support of C. Z. Serpan and Al Taboada of the Nuclear Regulatory Commission; E. Sajo and I. Remec for their suggestions in reviewing this report; L. P. Hileman for preparing the manuscript; and C. H. Shappert for editing. 


\section{INTRODUCTION}

Two carlier studies performed by the Oak Ridge National Laboratory (ORNL) for the U.S. Nuclear Regulatory Commission (NRC) have provided estimates for the neutron exposure to the reactor pressure vessel (RPV) of the H.B. Robinson (HBR) PWR. The first of these two studies compared results of transport calculations with measured activities of surveillance dosimeters located at both in vessel and reactor cavity positions during Cycle 9. The second ORNL study consisted of transport calculations to determine the fluence rate for HBR Cycle 10, which was the first cycle to utilize "partial length shield assemblics" (PLSAs) as a means of RPV flux reduction. In the latter study, the transport calculations were compared with experimental measurements of various dosimeters located only within the reactor cavity. ${ }^{2}$ In both of these earlier studics, inconsistencies were observed between the calculated and measured dosimeter activitics in the cavity region.

The Cycle 9 transport calculations were performed by Macrker and used the ELXSIR ${ }^{3}$ multigroup cross-section library, which is based on ENDF/B-IV data. A detailed sensitivity-uncertainty analysis using the LEPRICON ${ }^{1}$ system was performed as part of the study, and these calculations suggested that most of the observed discrepancies were due to the iron inelastic cross section in ENDF/B-IV, which causes high-energy threshold reactions to be underestimated in the cavity.

The Cycle 10 analysis was performed during 1989, approximately four years after the original Cycle 9 study. The Cycle 10 study used a modified version of the SAILOR cross-section library, ${ }^{4}$ which contained newer iron data based on a revised evaluation provided by $C$. Y. Fu of ORNL." This evaluation has been designated unofficially as the "REV.V" iron data. ${ }^{6}$ It was found that the REV.V iron cross sections used for the Cycle 10 transport calculations significantly increased the transmission of the fast flux through the HBR vessel compared with the ENDF/B-IV or ENDF/B.V iron evaluations, such as those evaluations used in the earlier Cycle 9 calculations. This REV.V iron cross section increases the calculated values for the higher threshold reactions, such as ${ }^{54} \mathrm{Fe}(\mathrm{n}, \mathrm{p})$ and ${ }^{58} \mathrm{Ni}(\mathrm{n}, \mathrm{p})$, and improves the agreement with the cavity measurements of these dosimeters. However, some inconsistencies still were unresolved, especially for the lower threshold ${ }^{237} \mathrm{~Np}(\mathrm{n}, \mathrm{f})$ dosimeter. It appears that the Fu REV.V iron evaluation does not significantly increase the calculated value for this dosimeter reaction, which unlike the iron and nickel dosimeters, is sensitive to the energy range between 0.5 and $2.0 \mathrm{MeV}$. Thus the computed neptunium activity remains substantially low relative to the experimental measurement while the iron and nickel value are computed much closer to the respective experimental results. This relationship suggests that the REV.V iron data may introduce a bias between the high-lhreshold and low-threshold reactions calculated in the cavity (which was not observed with the ENDF/B-IV or ENDF/B-V iron data). Because of increasing importance of cavity dosimetry in assessing pressure vessel fluence, it is very important to establish a reliable set of iron data that can be used as a standard for transport calculations. Neither the ENDF/B-IV or ENDF/B-V, which are the bases of the widely used SAILOR and ELXSIR multigroup libraries, nor the REV.V data utilized in the HBR Cycle 10 analysis, appears to be completely satisfactory in this regard.

In 1989, the most recent version of the ENDF/B was released by the National Nuclear Data Center (NNDC): ENDF/B-VI. This data file includes new iron cross-section evaluations that evolved from the carlier REV-V evaluation developed by Fu. Compared with earlier ENDF/B versions, the version VI iron evaluation gives a forward bias to the inelastic scatter kernel, and the magnitude of the inclastic cross section is reduced somewhat. Also, nuclear data are given for each of the iron isotopes, rather than for the composite "natural iron." These values allow for a more accurate treatment of the self-shiclding. These changes in the iron data will significantly impact the neutron transmission through the RPV. Preliminary results obtained in an carlier study suggest that ENDF/B-VI iron data will increase the computed dosimeter activities in the cavity of power reactors without introducing a bias between the high and lower threshold reaction rates, such as observed with the REV-V evaluation. ${ }^{6}$ However, this conclusion is based only on projections obtained from one-dimensional (1-D) calculations. No complete two-dimensiona! (2-D) calculation of a power reactor has been performed with ENDF/B-VI.

The objective of this study is to perform an updated calculation for H. B. Robinson Cycle 9 using selected ENDF/B-VI cross-section data. Specifically, the iron isotopes ${ }^{54} \mathrm{Fe},{ }^{56} \mathrm{Fe},{ }^{57} \mathrm{Fe}$, and ${ }^{58} \mathrm{Fe}$, and oxygen and hydrogen are all represented by ENDF/B.VI data. Data for all other materials are taken from the original SAILOR library, which is based on ENDF/B-IV. The results of this analysis will be important in assessing the merit of utilizing ENDF/B.VI for RPV fluence determination, and will help in deciding whether a new cross-section library based on 
ENDF/B-VI should replace the older libraries based on versions IV and $V$ that are widely used in industry today. These results are given in Section 3. This report also sumarizes results of a study aimed at understanding the differences observed in calculations performed with two commonly used cross-section libraries. The SAILOR and ELXSIR data librarics are used in many RPV fluence analysis studics, so it is important to understand any discrepancies that may be attributed to the libraries themselves. The impact of differences in self-shiclding assumptions and collapsing spectra curve are considered, in addition to the inherent variations due to different group structures. Spectral differences for both high threshold dosimeters like ${ }^{54} \mathrm{Fe}(\mathrm{n}, \mathrm{p})$ and ${ }^{58} \mathrm{Ni}(\mathrm{n}, \mathrm{p})$ and lower threshold dosimeters like ${ }^{277} \mathrm{~Np}(\mathrm{n}, \mathrm{f})$ and ${ }^{288} \mathrm{U}(\mathrm{n}, \mathrm{f})$ in a one-dimensional model of $\mathrm{H}$. B. Robinson are considered. These results are summarized in Section 4.

\section{CALCULATION METHODOLOGY}

The same method as used in the previous HBR transport calculations (and in many other studics) was also used in the current work. Basically, this consists of synthesizing a threc-dimensional (3-D) flux distribution from results obtained in 1- and 2-D DOT discrete-ordinates calculations. Details of these synthesis calculations are given in the references. ${ }^{1,2}$ The core source was based on the mid-cycle (MOC) distribution for Cycle 9. The spatial variation of the sourec is obtained by combining a pinwise relative power distribution with the absolute assembly-wise powers to determine the absolute pin-wise power at MOC. This distribution is mapped onto a $R \theta$ coordinate system used in the transport calculations. The conversion factor from power density to neutron source density is based on the average burnup of the peripheral assemblies in order to reflect the proper contributions of ${ }^{239} \mathrm{Pu}$ and ${ }^{235} \mathrm{U}$ to the fission source. Similarly, the fission spectrum is a weighted combination of these two isotopes at the appropriate burnup condition. Extension of the mic-cycle calculations to end of eycle (EOC) was done by multiplying by EOC-to-MOC ratios reported by Maerker ${ }^{1}$ in his carlier Cycle 9 analysis.

The transport model used in this analysis is virtually identical to that used in Macrker's earlier Cycle 9 analysis, except near the $45^{\circ}$ azimuth, where an additional "detector well" is included within the cavity region. Figure 2.1 shows the R $\theta$ model of the one-cighth HBR core. Note the presence of the two large cavity "wells" within the concrete center wall of the cavity at $0^{\circ}$ and $45^{\circ}$. The wells affect the flux spectrum near these locations and cause difficulty in utilizing 1-D or RZ calculations to estimate the flux spectrum in the cavity, because the wells cannot be represented with axisymmetric geometries.

The cross-section library used in the calculations was a modified version of the 47-neutron group SAlLOR library. The original SAILOR library was collapsed from the ENDF/B-IV VITAMIN.C library, ${ }^{7}$ the same fine-group library that was collapsed to create the 56-group ELXSIR library used in Macrker's earlier analysis of the HBR Cycle 9 dosimetry. The modified SAILOR library contains additional cross sections for iron, oxygen, and hydrogen $(\mathrm{Fe}-\mathrm{O} \cdot \mathrm{H})$ that were processed from ENDF/B-VI data by J. E. White of ORNL, using the same processing methodology as for the original SAILOR. Calculations were performed with both the original SAILOR and the ENDF/B-VI Fc-O.H cross sections, for comparison with the earlier results obtained with ELXSIR.

Figure 2.2 shows the ratios of the iron inclastic cross section in REV.V and ENDF/B.VI, respectively, to the ENDF/B-V values (which are essentially the same as version IV in the energy range of interest here). The ENDF/B-VI data are about $10 \%$ lower than ENDF/B-V cross sections between 0.5 and $0.86 \mathrm{McV}$, and are up to $14 \%$ lower in the energy range between 2.5 and $6.0 \mathrm{MeV}$. The REV.V inclastic data are also much lower than ENDF/B-V hetween 3.5 and $6.0 \mathrm{MeV}$ (even more so than version VI); however, there is no difference between the REV.V and ENDF/B.V helow $2.5 \mathrm{McV}$. The cross sections in the higher energy interval affect the neutron spectrum in the range to which the ${ }^{54} \mathrm{Fe}(n, p)$ and ${ }^{58} \mathrm{Ni}(n, p)$ threshold reactions are most sensitive, whereas the ${ }^{237} \mathrm{~Np}(\mathrm{n}, \mathrm{f})$ is sensitive to the energy range from 0.5 to $2.0 \mathrm{McV}$. The differences in the REV - V and ENDF/B.VI inclastic cross section in this lower energy interval will cause the two data files $(0)$ affect the calculated $\mathrm{Np}$ reaction rate differently.

Because of the close similarity between the present transport calculations and those in the early Cycle 9 analysis, the difference observed in the results from the two studies will reflect primarily the impact of version VI versus IV nuclear data. However, variations in the multigroup processing procedures and group structures of the SAILOR and ELXSIR cross-section lihraries will also cause some discrepancies. A separate study using 1-D calculations 
was performed to investigate the effect of various resonance self-shiclding, cross-section weighting spectra, and group-structure differences on the computed results. The one-dimensional model used in the transport calculations was the same as used in the synthesis calculation.

ORNL DWG. NO. 89-19727

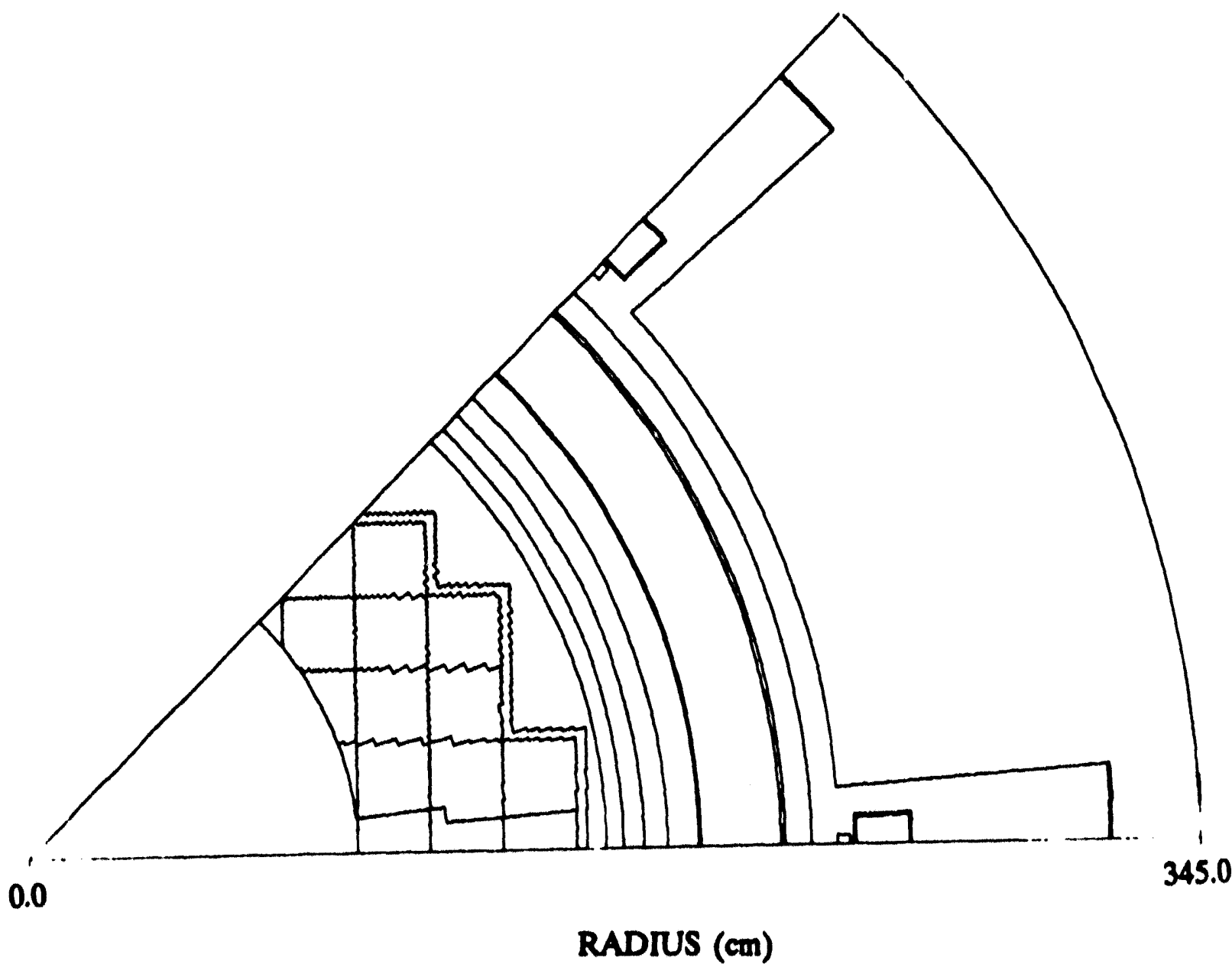

Figure 2.1 DOT RO model of H. B. Robinson Unit 2 


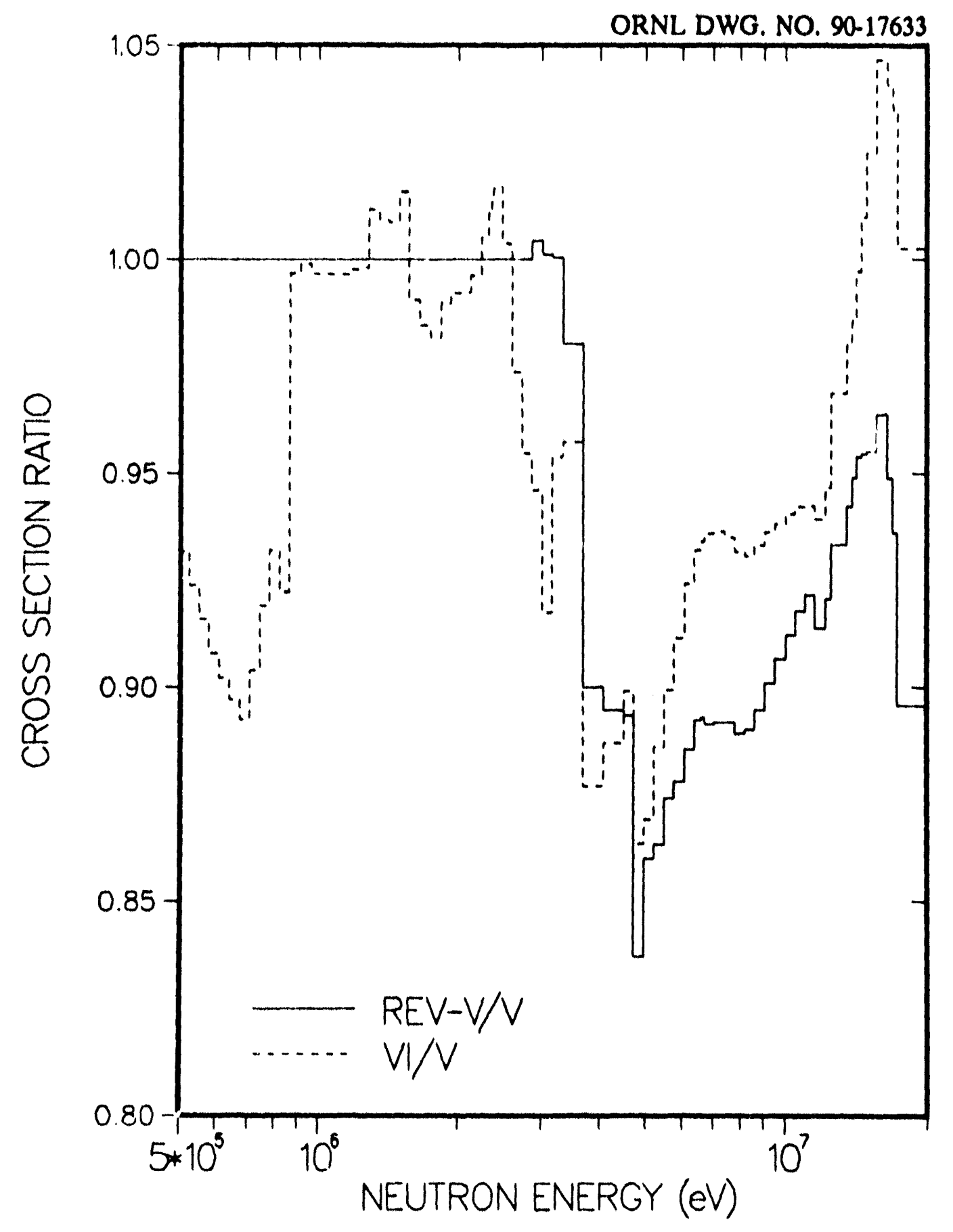

Figure 2.2 Ratio of REV-V and ENDF/B-VI inclastic multigroup cross sections to the ENDF/B-V values (natural iron). 


\section{RESULTS FOR HBR CYCLE?}

Tables 3.1 and 3.2, respectively, show the absolute flux spectra at the surveillance location $\left(20^{\circ}\right)$ in the downconer and at the cavity location $\left(0^{\circ}\right)$ obtained with the modified SAILOR library which contains ENDF/B.VI Fc-O-H data. The absolute dosimeter reaction rates computed with these spectra are given in Table 3.3. The values in Table 3.3 were obtained with the ENDF/B.VI file. In calculating the dosimeter activities, two differetil sets of dosimetry cross sections were used. One is based on the cross sections taken from ENDF/B.VI. The other, designated as the "original desimetry file," corresponds to data taken from the LSLM-2 adjustment code, file CO1. The major difference is in the ${ }^{63} \mathrm{Cu}(n, a)$ reaction, which is known to be loo large in the carlier dosimeiry lile.

Table 3.4 compares our results computed using the original SAILOR library with Macrker's carlier values calculated with ELXSIR cross sections. Recall that both of these libraries are based on the same ENDI; B-IV data and should be expected to give similar results. In the downcomer region, the original SAILOR and the E:LXSIR results agree well using either set of dosimetry data, except for the ${ }^{63} \mathrm{Cu}(\mathrm{n}, \alpha)$ value which is overpredicted by about $22 \%$ with the original dosimeter cross section data. This discrepancy in the carlier ${ }^{63} \mathrm{Cu}$ desimetry data is well known and explains this diffcrence. However, something strange happens in the cavity region: the original SAILOR calculations with both dosimetry files give about a 20 to $25 \%$ lower value than Macrker's ELXSIR results for the ${ }^{277} \mathrm{~Np}$ reaction. All other threshold reactions computed with the original SAILOR and the ELXSIR libraries agree well. This variation suggests that the neutron spectra calculated with the two respective libraries for the cavity are significantly different in the energy range below $2.0 \mathrm{McV}$, even though both librarics are based on ENDF/B-IV. As discussed in the next section, there appears to be about a $20 \%$ discrepancy in the flux above $0.1 \mathrm{McV}$ calculated with ELXSIR and the original SAILOR, respectively, that is presumably caused by the coarser group structure in SAILOR. ELXSIR agrees better with fine-group results, which are assumed to be the most accurate calculations.

Tables 3.5 and 3.6 show that the ENDF/B-VI Fe-O-H increase the original SAILOR values of the calculated dosimeter activities in the cavity by about 30 to $35 \%$, except for the very high-threshold ${ }^{6} \mathrm{Cu}(\mathrm{n}, \alpha)$, which only increases about $20 \%$. This dosimeter behaves differenily than the others because of its higher sensitivity in the energy range above $10.0 \mathrm{MeV}$, where the ENDF/B.VI iron inclastic cross section does not decrease as much compared with the earlicr ENDF versions. The cross section around $14.0 \mathrm{McV}$ is actually higher in version $\mathrm{VI}$ than version $V$ (see Fig. 2.2). These changes for the various dosimeters are more uniform and consistent than found earlier for the Cycle 10 analysis where the REV.V iron data were used. For cxample, it was found that the REV.V data increased the ${ }^{54} \mathrm{Fe}$ dosimeter activity by $60 \%$ and the ${ }^{217} \mathrm{~Np}$ activity by only $28 \%$, whereas the ENDF/B.VI data increased the two dosimeter activitics by $35 \%$ and $32 \%$, respectively.

Finally, the revised C/E values obtained for HBR Cycle 9 are shown in Table 3.7. Experimental values were obtained from reference 9. Computed activities in the cavity region are significantly underestimated with both the ELXSIR and original SAILOR libraries. However, it can be seen that the ENDF/B.VI results show excellent agreement with measured dosimeter results at both the downeomer and cavity locations. Most C/E's are within 5\% of unity. A notable exception is the cavity ${ }^{237} \mathrm{~Np}$ value, which is still not consistent with the other values. Based on the analysis described in the next section, it is felt that the SAILOR calculation of the ${ }^{217} \mathrm{~Np}$ dosimeter is probably about 10 to $15 \%$ too low due to group structures and/or multigroup processing approximations. Even after applying this correction, the neptunium $\mathrm{C} / \mathrm{E}$ value will remain about $30 \%$ lower than the other dosimeter results. We feel that this residual discrepancy is probably caused by a measurement problem in the HBR Cycle 9 experiment for the neptunium dosimeter.

The new HBR results are consistent with earlier studics that projected that ENDF/B-VI iron cross sections would significantly improve the calculations of cavity dosimetry. ${ }^{6}$ If other power reactors behave similarly to HBR, then it appears that ENDF/B-VI cross sections should replace the older library data currently in use.

The next section examines the impact of various cross-section libraries on RPV dosimeter calculations. 
Table 3.1 Absolute spectra at $20^{\circ}$ surveillance location in HBR Cycle 9 (calculated with ENDF/B-VI Fe-O-II)

\begin{tabular}{|c|c|c|c|c|c|c|}
\hline Group & $\begin{array}{l}\text { Upper } \\
\text { Energy } \\
(\mathrm{MeV})\end{array}$ & $\begin{array}{l}\text { Group } \\
\text { nux } \\
\left(\mathrm{n} \cdot \mathrm{cm}^{-2} \cdot \mathrm{s}^{-1}\right)\end{array}$ & $\begin{array}{l}\text { Cumulative } \\
\text { flux } \\
\left(\mathrm{n} \cdot \mathrm{cm}^{-2} \cdot \mathrm{s}^{-1}\right)\end{array}$ & $\begin{array}{l}\text { Group } \\
\text { dpa }\end{array}$ & $\begin{array}{c}\text { Cumulative } \\
\text { dpa }\end{array}$ & $\begin{array}{c}\text { dpa } \\
\text { fraction* }\end{array}$ \\
\hline 1 & $1.733 \mathrm{E}+01$ & $6.078 \mathrm{E}+06$ & $6.078 \mathrm{E}+06$ & 1.776E:-14 & $1.776 \mathrm{E}-14$ & $3.225 \mathrm{E}-04$ \\
\hline 2 & $1.419 \mathrm{E}+01$ & $2445 \mathrm{E}+07$ & $3.053 E+07$ & $6.469 \mathrm{IL}-14$ & 8.245E-14 & $1.497 \mathrm{E}-03$ \\
\hline 3 & $1.221 \mathrm{E}+01$ & $9.620 \mathrm{E}+07$ & $1.267 \mathrm{E}+08$ & 2.317E-13 & $3.142 \mathrm{E}-13$ & $5.706 \mathrm{E}-03$ \\
\hline 4 & $1.000 \mathrm{E}+01$ & $1.978 \mathrm{E}+08$ & $3.245 \mathrm{E}+08$ & $4.389 \mathrm{E}-13$ & $7.531 \mathrm{E}-13$ & $1.368 \mathrm{E}-02$ \\
\hline 5 & $8.607 \mathrm{E}+00$ & $3.443 E+08$ & $6.688 \mathrm{E}+08$ & $7.185 \mathrm{E}-13$ & 1.472E-12 & 2673E-02 \\
\hline 6 & $7.408 \mathrm{E}+00$ & $8.333 E+08$ & $1.502 \mathrm{E}+09$ & $1.619 \mathrm{E}-12$ & $3.091 \mathrm{E}-12$ & 5.613E-02 \\
\hline 7 & $6.065 \mathrm{E}+00$ & $1.232 \mathrm{E}+09$ & $2734 \mathrm{E}+09$ & 2.198E-12 & $5.289 \mathrm{E}-12$ & $9.605 \mathrm{E}-02$ \\
\hline 8 & $4.966 \mathrm{E}+00$ & $2373 \mathrm{E}+09$ & $5.107 \mathrm{E}+09$ & 3.728E-12 & $9.016 \mathrm{E}-12$ & $1.637 \mathrm{E}-01$ \\
\hline 9 & $3.679 \mathrm{E}+00$ & $1.845 \mathrm{E}+09$ & $6.952 \mathrm{E}+09$ & 2.528E-12 & $1.154 \mathrm{E}-11$ & 2.097E-01 \\
\hline 10 & $3.012 \mathrm{E}+00$ & $1.404 \mathrm{E}+09$ & $8.356 \mathrm{E}+09$ & $1.784 \mathrm{E}-12$ & 1.333E-11 & 2.421E-01 \\
\hline 11 & $2.725 \mathrm{E}+00$ & $1.653 \mathrm{E}+09$ & $1.001 \mathrm{E}+10$ & $2.108 \mathrm{E}-12$ & $1.544 \mathrm{E}-11$ & 2.803E-01 \\
\hline 12 & $2466 \mathrm{E}+00$ & $8.123 E+08$ & $1.082 \mathrm{E}+10$ & $9.520 \mathrm{E}-13$ & 1.639E-11 & 2.976E-01 \\
\hline 13 & $2365 E+00$ & $2338 \mathrm{E}+08$ & $1.106 \mathrm{E}+10$ & $2.562 \mathrm{E}-13$ & $1.664 \mathrm{E}-11$ & $3.023 \mathrm{E}-01$ \\
\hline 14 & $2.346 \mathrm{E}+00$ & $1.144 \mathrm{E}+09$ & $1.220 \mathrm{E}+10$ & $1.191 \mathrm{E}-12$ & $1.784 \mathrm{E}-11$ & $3.239 \mathrm{E}-01$ \\
\hline 15 & $2.231 E+00$ & $3.124 \mathrm{E}+09$ & $1.532 \mathrm{E}+10$ & $3.230 \mathrm{E}-12$ & 2.107E-11 & $3.826 \mathrm{E}-01$ \\
\hline 16 & $1.920 \mathrm{E}+00$ & $3.719 \mathrm{E}+09$ & $1.904 \mathrm{E}+10$ & $3.025 \mathrm{E}-12$ & $2.409 \mathrm{E}-11$ & 4.375E 01 \\
\hline 17 & $1.653 \mathrm{E}+00$ & $5.420 \mathrm{E}+09$ & $2446 \mathrm{E}+10$ & $4.392 E-12$ & 2.848E-11 & 5.173E-01 \\
\hline 18 & $1.353 E+00$ & $9.931 \mathrm{E}+09$ & $3.439 \mathrm{E}+10$ & $5.565 \mathrm{E}-12$ & 3.405E-11 & 6.183E-01 \\
\hline 19 & $1.003 \mathrm{E}+00$ & $6.900 \mathrm{~L}+09$ & $4.129 \mathrm{E}+10$ & 2.52.9Г- 12 & 3.658E-11 & $6.643 E-01$ \\
\hline 20 & 8.208E-01 & $3.349 \mathrm{E}+09$ & $4.464 \mathrm{E}+10$ & $1.879 \mathrm{E}-12$ & $3.845 \mathrm{E}-11$ & $6.984 \mathrm{E}-01$ \\
\hline 21 & 7.427E-01 & $9.370 E+09$ & $5.401 \mathrm{E}+10$ & $3.3866 \mathrm{E}-12$ & $4.184 \mathrm{E}-11$ & $7.599 \mathrm{E}-01$ \\
\hline 22 & $6.081 \mathrm{E}-01$ & $7.901 \mathrm{E}+09$ & $6.191 \mathrm{E}+10$ & 2.325E-12 & 4.417E-11 & 8.021E-01 \\
\hline 23 & 4.979E-01 & $8.370 \mathrm{E}+09$ & $7.029 \mathrm{~L}+10$ & 3.318E-12 & $4.748 \mathrm{E}-11$ & 8.624E-01 \\
\hline 24 & $3.688 \mathrm{E}-01$ & $7.584 \mathrm{E}+09$ & $7.787 \mathrm{E}+10$ & $1.568 \mathrm{E}-12$ & $4.905 \mathrm{E}-11$ & 8.908E-01 \\
\hline 25 & 2972E-01 & $1.121 \mathrm{E}+10$ & $8.908 \mathrm{E}+10$ & $2.248 \mathrm{E}-12$ & $5.130 \mathrm{E}-11$ & 9.317R-01 \\
\hline 26 & $1.832 \mathrm{E}-01$ & $9.518 \mathrm{E}+09$ & $9.860 \Gamma+10$ & $1.342 \mathrm{E}-12$ & $5.264 \mathrm{E}-11$ & $9.560 \mathrm{E}-01$ \\
\hline 27 & $1.111 \mathrm{E}-01$ & $7.375 \mathrm{E}+09$ & $1.060 \mathrm{E}+11$ & $9.536 \mathrm{E}-13$ & $5.360 \mathrm{E}-11$ & $9.734 \mathrm{E}-01$ \\
\hline 28 & $6.738 \mathrm{E}-02$ & $6.268 \mathrm{E}+09$ & $1.122 \mathrm{E}+11$ & 4.060E-13 & $5.400 \mathrm{E}-11$ & $9.807 \mathrm{E}-01$ \\
\hline 29 & $4.087 \mathrm{E}-02$ & $2409 \mathrm{E}+09$ & $1.147 \mathrm{E}+11$ & $1.949 \mathrm{E}-13$ & $5.420 \mathrm{E}-11$ & 9.843E-01 \\
\hline 30 & $3.183 \mathrm{E}-02$ & $1.178 \mathrm{E}+09$ & $1.158 \mathrm{E}+11$ & $3.349 \mathrm{E}-13$ & $5.453 \mathrm{E}-11$ & $9.904 \mathrm{E}-01$ \\
\hline 31 & $2606 \mathrm{E}-02$ & $2.1511+09$ & $1.180 \mathrm{E}+11$ & 4.337E-14 & $5.458 \mathrm{E}-11$ & $9.911 \mathrm{E}-01$ \\
\hline 32 & 2418E-02 & $1.272 \mathrm{E}+09$ & $1.193 \mathrm{E}+11$ & $5.571 \mathrm{E}-15$ & $5.458 \mathrm{E}-11$ & $9.912 \mathrm{E}-01$ \\
\hline 33 & 2.188E-02 & $3.426 \mathrm{E}+09$ & $1.227 \mathrm{E}+11$ & 2.806[:-14 & $5.461 \mathrm{E}-11$ & $9.918 \mathrm{E}-01$ \\
\hline 34 & $1.503 E-02$ & $6.526 \mathrm{E}+09$ & $1.292 \mathrm{E}+11$ & $1.224 \mathrm{E}-13$ & $5.473 \mathrm{E}-11$ & $9.940 \mathrm{E}-01$ \\
\hline 35 & $7.102 \mathrm{E}-03$ & $7.034 \mathrm{E}+09$ & $1.362 \mathrm{IE}+11$ & 6.257E:-14 & $5.479 \mathrm{E}-11$ & 9.951E-01 \\
\hline 36 & 3.355E-03 & $6.511 \mathrm{E}+09$ & $1.427 \mathrm{E}+11$ & 2.292E:-14 & $5.482 \mathrm{E}-11$ & 9.955E-01 \\
\hline 37 & $1.585 \mathrm{E}-03$ & $1.094 \mathrm{E}+10$ & $1.5371 \mathrm{i}+11$ & $1.840 \mathrm{E}-14$ & $5.484 \mathrm{E}-11$ & 9.959E-01 \\
\hline 38 & $4.540 \mathrm{E}-04$ & $5.733 E+09$ & $1.594 \mathrm{E}+11$ & $5.652 \mathrm{E}-16$ & $5.484 \mathrm{E}-11$ & 9.959E-01 \\
\hline 39 & 2.144E-04 & $6.400 \mathrm{E}+09$ & $1.658 \mathrm{E}+11$ & 9.177E-16 & $5.484 \mathrm{E}-11$ & 9.959E-01 \\
\hline 40 & 1.013E-04 & $8.475 E+09$ & $1.743 E+11$ & $1.891 \mathrm{E}-15$ & $5.484 \mathrm{E}-11$ & 9.959E-01 \\
\hline 41 & 3.727E-05 & $1.038 \mathrm{E}+10$ & $1.847 \mathrm{E}+11$ & $4.088 \mathrm{E}-15$ & 5.484E-11 & 9.960E-01 \\
\hline 42 & $1.068 \mathrm{E}-05$ & $5.9441:+09$ & $1.906 \mathrm{E}+11$ & $3.821 \mathrm{E}-15$ & $5.485 \mathrm{E}-11$ & $9.961 \mathrm{E}-01$ \\
\hline 43 & $5.043 E-06$ & $7.4141 \mathrm{I}+\infty 9$ & $1.980 \mathrm{I}+11$ & $7.414 \mathrm{~L}-15$ & $5.485 \mathrm{E}-11$ & 9.962E-01 \\
\hline 44 & $1.855 \mathrm{E}-06$ & $4.935 \mathrm{E}+09$ & $2030 \mathrm{E}+11$ & $7.5851 \mathrm{~L}-15$ & $5.486 \mathrm{E}-1 \mathrm{i}$ & $9.963 \mathrm{E}-01$ \\
\hline 45 & $8.764 \mathrm{E}-07$ & $4.435 E+09$ & $2.074 \mathrm{~L}+11$ & $9.952 \mathrm{~L}-1.5$ & $5.487 \mathrm{E}-11$ & $9.965 \mathrm{E}-01$ \\
\hline 46 & 4.1401:-07 & $4.520 \mathrm{~L}+09$ & $2119 E+11$ & 1.771E:-14 & 5.489E-11 & $9.968 \mathrm{E}-01$ \\
\hline 47 & $1.000 \mathrm{E}-07$ & $1.857 \mathrm{E}+10$ & $2.305 E+11$ & $1.736 \mathrm{~L}:-13$ & $5.506 \mathrm{E}-11$ & $1.000 \mathrm{E}+00$ \\
\hline
\end{tabular}

- Cumulative dpa normalized to 1.0

* Lower energy boundary for group $47=1.0 \mathrm{E}-11$ 
Table 3.2 Absolute spectra at $0^{\circ}$ cavity location in HBR Cycle 9 (calculated with ENDF/B-VI Fe-O-H)

\begin{tabular}{|c|c|c|c|c|c|c|}
\hline Group & $\begin{array}{l}\text { Upper } \\
\text { Energy } \\
(\mathrm{MeV})\end{array}$ & $\begin{array}{l}\text { Group } \\
\text { flux } \\
\left(n \cdot \mathrm{cm}^{-2} \cdot \mathrm{s}^{-1}\right)\end{array}$ & $\begin{array}{l}\text { Cumulative } \\
\text { flux } \\
\left(\mathrm{n} \cdot \mathrm{cm}^{-2} \cdot \mathrm{s}^{-1}\right)\end{array}$ & $\begin{array}{l}\text { Group } \\
\text { dpa }\end{array}$ & $\begin{array}{c}\text { Cumulative } \\
\text { dpa }\end{array}$ & $\begin{array}{c}\text { dpa } \\
\text { fraction* }\end{array}$ \\
\hline 1 & $1.733 E+01$ & $9.219 \mathrm{E}+04$ & $9.219 \mathrm{E}+04$ & $2694 \mathrm{E}-16$ & 2.694E-16 & $1.058 \mathrm{E}-04$ \\
\hline 2 & $1.419 \mathrm{E}+01$ & $3.544 \mathrm{E}+05$ & $4.466 \mathrm{E}+05$ & $9.377 \mathrm{E}-16$ & 1.207E-15 & $4.741 \mathrm{E}-04$ \\
\hline 3 & $1.221 \mathrm{E}+01$ & $1.237 \mathrm{E}+06$ & $1.684 \mathrm{E}+06$ & $2980 \mathrm{E}-15$ & 4.187E-15 & $1.644 \mathrm{E}-03$ \\
\hline 4 & $1.000 \mathrm{E}+01$ & $2.342 \mathrm{E}+06$ & $4.026 \mathrm{E}+06$ & 5.197E-15 & $9.384 \mathrm{E}-15$ & $3.685 \mathrm{E}-03$ \\
\hline 5 & $8.607 \mathrm{E}+00$ & $3.542 \mathrm{E}+06$ & $7.567 \mathrm{E}+06$ & $7.391 \mathrm{E}-15$ & $1.678 \mathrm{E}-14$ & $6.588 \mathrm{E}-03$ \\
\hline 6 & $7.408 \mathrm{E}+00$ & $7.086 \mathrm{E}+06$ & $1.465 E+07$ & 1.377E-14 & $3.054 \mathrm{E}-14$ & $1.199 \mathrm{E}-02$ \\
\hline 7 & $6.065 E+00$ & $9.813 E+06$ & $2.447 E+07$ & $1.751 \mathrm{E}-14$ & $4.805 \mathrm{E}-14$ & $1.887 \mathrm{E}-02$ \\
\hline 8 & $4.966 \mathrm{E}+00$ & $1.887 \mathrm{E}+07$ & $4.333 E+07$ & 2964E-14 & $7.769 \mathrm{E}-14$ & 3.051E-02 \\
\hline 9 & $3.679 \mathrm{E}+00$ & $1.599 \mathrm{E}+07$ & $5.933 \mathrm{E}+07$ & 2.191E-14 & $9.960 \mathrm{E}-14$ & $3.912 \mathrm{E}-02$ \\
\hline 10 & $3.012 \mathrm{E} \div 00$ & $1.316 \mathrm{E}+07$ & $7.249 \mathrm{E}+07$ & $1.673 \mathrm{E}-14$ & 1.163E-13 & $4.568 \mathrm{E}-02$ \\
\hline 11 & $2.725 E+\infty 0$ & $1.666 \mathrm{E}+07$ & $8.915 \mathrm{E}+07$ & $2.124 \mathrm{E}-14$ & $1.376 \mathrm{E}-13$ & $5.403 \mathrm{E}-02$ \\
\hline 12 & $2466 \mathrm{E}+00$ & $8.788 E+06$ & $9.793 E+07$ & $1.030 \mathrm{E}-14$ & $1.479 \mathrm{E}-13$ & $5.807 \mathrm{E}-02$ \\
\hline 13 & $2365 \mathrm{E}+00$ & $2.975 E+06$ & $1.009 \mathrm{E}+08$ & $3.260 \mathrm{E}-15$ & $1.511 \mathrm{E}-13$ & $5.935 \mathrm{E}-02$ \\
\hline 14 & $2346 \mathrm{E}+00$ & $1.498 \mathrm{E}+07$ & $1.159 E+08$ & 1.559E-14 & $1.667 \mathrm{E}-13$ & $6.547 \mathrm{E}-02$ \\
\hline 15 & $2.231 E+\infty 0$ & $4.148 E+07$ & $1.574 \mathrm{E}+08$ & $4.289 \mathrm{E}-14$ & $2.096 \mathrm{E}-13$ & $8.232 E-02$ \\
\hline 16 & $1.920 \mathrm{E}+00$ & $6.640 \mathrm{E}+07$ & $2.238 E+08$ & $5.400 \mathrm{E}-14$ & $2.636 \mathrm{E}-13$ & $1.035 \mathrm{E}-01$ \\
\hline 17 & $1.653 \mathrm{E}+00$ & $1.125 \mathrm{E}+08$ & $3.363 \mathrm{E}+08$ & $9.120 \mathrm{E}-14$ & $3.548 \mathrm{E}-13$ & $1.393 \mathrm{E}-01$ \\
\hline 18 & $1.353 E+00$ & $3.094 \mathrm{E}+08$ & $6.457 \mathrm{E}+08$ & $1.734 \mathrm{E}-13$ & $5.282 \mathrm{E}-13$ & $2.074 \mathrm{E}-01$ \\
\hline 19 & $1.003 E+00$ & $3.2261 \mathrm{I}+08$ & $9.683 \mathrm{E}+08$ & $1.182 \mathrm{E}-13$ & $6.464 \mathrm{~L}-13$ & 2.539E-01 \\
\hline 20 & 8.208E-01 & $1.426 \mathrm{E}+08$ & $1.111 \mathrm{E}+09$ & 7.997E-14 & $7.264 \mathrm{E}-13$ & 2.853E-01 \\
\hline 21 & 7.427E-01 & $8.286 \mathrm{E}+08$ & $1.940 \mathrm{E}+09$ & 2.995E-13 & $1.026 \mathrm{E}-12$ & $4.029 \mathrm{E}-01$ \\
\hline 22 & 6.081E-01 & $7.311 \mathrm{E}+08$ & $2671 E+09$ & $2.151 \mathrm{E}-13$ & $1.241 \mathrm{E}-12$ & 4.874E-01 \\
\hline 23 & 4.9791E-01 & $8.233 E+08$ & $3.494 \mathrm{E}+09$ & $3.264 \mathrm{E}-13$ & $1.567 \mathrm{E}-12$ & $6.155 \mathrm{E}-01$ \\
\hline 24 & $3.688 \mathrm{E}-01$ & $1.245 \mathrm{E}+09$ & $4.738 \mathrm{E}+09$ & $2574 \mathrm{E}-13$ & $1.825 \mathrm{E}-12$ & 7.166E-01 \\
\hline 25 & 2.972E-01 & $1.462 \mathrm{E}+09$ & $6.200 E+09$ & $2.931 \mathrm{E}-13$ & 2.118E-12 & 8.317E-01 \\
\hline 26 & $1.832 \mathrm{E}-01$ & $1.355 \mathrm{E}+09$ & $7.555 \mathrm{E}+09$ & $1.910 \mathrm{E}-13$ & 2.309E-12 & 9.067E-01 \\
\hline 27 & $1.111 \mathrm{E}-01$ & $8.587 E+08$ & $8.414 E+09$ & 1.110E:-13 & $2420 \mathrm{E}-12$ & 9.503E-01 \\
\hline 28 & 6.738E-02 & $5.844 \mathrm{E}+08$ & $8.998 \mathrm{E}+09$ & $3.785 \mathrm{E}-14$ & $2458 \mathrm{E}-12$ & 9.652E-01 \\
\hline 29 & 4.087E-02 & $1.982 \mathrm{E}+08$ & $9.197 \mathrm{E}+09$ & $1.604 \mathrm{E}-14$ & $2.474 \mathrm{E}-12$ & 9.715E-01 \\
\hline 30 & 3.183E-02 & $1.209 \mathrm{E}+08$ & $9.317 \mathrm{E}+09$ & $3.437 E-14$ & $2.508 \mathrm{E}-12$ & $9.850 \mathrm{E}-01$ \\
\hline 31 & $2.606 \mathrm{E}-02$ & $4.346 E+08$ & $9.752 \mathrm{E}+09$ & 8.761L-15 & 2.517E-12 & 9.884E-01 \\
\hline 32 & 2418E-02 & $2.587 \mathrm{E}+08$ & $1.001 \mathrm{E}+10$ & $1.133 \mathrm{~L}-15$ & 2518E-12 & $9.889 \mathrm{E}-01$ \\
\hline 33 & 2.188E-02 & $4.159 \mathrm{E}+08$ & $1.043 \mathrm{E}+10$ & $3.406 \mathrm{I}-15$ & $2521 \mathrm{E}-12$ & $9.902 \mathrm{E}-01$ \\
\hline 34 & $1.503 \mathrm{E}-02$ & $5.199 \mathrm{E}+08$ & $1.095 E+10$ & $9.754 \mathrm{E}-15$ & $2.531 \mathrm{E}-12$ & $9.941 \mathrm{E}-01$ \\
\hline 35 & 7.102E-03 & $4.995 \mathrm{E}+08$ & $1.145 E+10$ & 4.443E-15 & 2536L-12 & 9.958E-01 \\
\hline 36 & 3.355E-03 & $4.213 E+08$ & $1.187 \mathrm{E}+10$ & $1.483 \mathrm{E}-15$ & $2537 \mathrm{E}-12$ & 9.964E-01 \\
\hline 37 & $1.585 \mathrm{E}-03$ & $6.1341 \mathrm{I}+08$ & $1.248 \mathrm{E}+10$ & $1.032 \mathrm{E}-15$ & $2.538 \mathrm{E}-12$ & $9.968 \mathrm{E}-01$ \\
\hline 38 & 4.540E-04 & $3.002 \mathrm{E}+08$ & $1.278 \mathrm{I}:+10$ & 2.959L-17 & $2.538 \mathrm{E}-12$ & $9.968 \mathrm{E}-01$ \\
\hline 39 & 2.144E-04 & $2.930 \mathrm{E}+08$ & $1.307 \mathrm{E}+10$ & $4.202 \mathrm{E}-17$ & 2538E-12 & $9.968 \mathrm{E}-01$ \\
\hline 40 & $1.013 E-04$ & $3.590 \mathrm{E}+08$ & $1.343 E+10$ & 8.010I:-17 & 2538E-12 & $9.9 \times 8 \mathrm{E}-01$ \\
\hline 41 & 3.727E-05 & $4.006 \mathrm{E}+08$ & $1.383 E+10$ & $1.578 \mathrm{E}-16$ & 2.539L-12 & 9.959E-01 \\
\hline 42 & 1.068E-05 & $2.146 \mathrm{E}+08$ & $1.405 \mathrm{E}+10$ & 1.379I:-16 & 2539L- 12 & 9.970E-01 \\
\hline 43 & 5.043E-06 & $2.439 \mathrm{E}+08$ & $1.429 \mathrm{E}+10$ & $2439 \mathrm{E}-16$ & 2.539IE-12 & $9.971 \mathrm{E}-01$ \\
\hline 44 & 1.855E-06 & $1.521 \mathrm{E}+08$ & $1.444 \mathrm{E}+10$ & $2.338 \mathrm{E}:-16$ & $2.539 \mathrm{E}-12$ & $9.972 \mathrm{E}-01$ \\
\hline 45 & 8.764Г-07 & $1.273 E:+08$ & $1.457 \mathrm{E}+10$ & 2856E:-16 & 2.539L- 12 & $9.973 E-01$ \\
\hline 46 & 4.140I:-07 & $1.277 \mathrm{E}+08$ & $1.470 \mathrm{I}+10$ & $5.003 E-16$ & $2.540 \mathrm{I}-12$ & 9.975E-01 \\
\hline 47 & 1.000I-07 & $6.919 \mathrm{E}+08$ & $1.539 \mathrm{E}+10$ & $6.468 \mathrm{~L}:-15$ & $2.546 \mathrm{~L}-12$ & $1.000 \mathrm{E}+00$ \\
\hline
\end{tabular}

- Cumulative dpa normalized to 1.0

* Iower cnergy boundary for group $47=1.0 \mathrm{E}$-11 
Table 33 Absolute dosimeter response rates for mid-cycle 9 of IIBR (calculated with ENDF/B-VI Fe-O-H and Version VI dosimeter cross sections)

\begin{tabular}{|c|c|c|}
\hline Reactions & Surveillance & Cavity \\
\hline${ }^{s+} \operatorname{Fc}(n, p)$ & $2.9071 \mathrm{~L}-15$ & $2690 \mathrm{E}-17$ \\
\hline${ }^{58} \mathrm{Ni}(\mathrm{n}, \mathrm{p})$ & $3.932 \mathrm{E}-15$ & $4.008 \mathrm{E}-17$ \\
\hline${ }^{\infty} \mathrm{Cu}(\mathrm{n}, \alpha)$ & 2.951E-17 & 2.997E-19 \\
\hline${ }^{27} \mathrm{~Np}(\mathrm{n}, \mathrm{I})$ & 8.749E-14 & $3.380 \mathrm{E}-15$ \\
\hline${ }^{208} \mathrm{U}(\mathrm{n}, \mathrm{n})$ & $1.285 \mathrm{E}-14$ & $1.793 \mathrm{E}-16$ \\
\hline${ }^{*} \mathrm{Ti}(\mathbf{n}, \mathbf{p})$ & 4.649E-16 & $4.274 \mathrm{E}-18$ \\
\hline Flux $>1.0 \mathrm{MeV}$ & $3.450 E+10$ & $6.509 \mathrm{E}+08$ \\
\hline Flux $>0.1 \mathrm{McV}$ & $9.860 \mathrm{E}+10$ & $7.555 \mathrm{E}+09$ \\
\hline
\end{tabular}

Table 3.4 Ratio of dosimeter reaction rates obtained with original SAILOR Library to valucs obtaincd with ELXSIR

\begin{tabular}{|c|c|c|c|c|}
\hline \multirow[b]{2}{*}{ Dosimetcr } & \multicolumn{2}{|c|}{$\begin{array}{r}\text { ENDF/B-VI } \\
\text { dosimetry filc }\end{array}$} & \multicolumn{2}{|c|}{$\begin{array}{c}\text { Original } \\
\text { dosimetry file }\end{array}$} \\
\hline & Downcomer* & Cavity** & Downcomcr * & Cavity** \\
\hline${ }^{54} \mathrm{Fc}(\mathrm{n}, \mathrm{p})$ & 1.06 & 1.06 & 1.06 & 1.06 \\
\hline${ }^{58} \mathrm{Ni}(\mathrm{n}, \mathrm{p})$ & 1.09 & 1.10 & 1.06 & 1.12 \\
\hline${ }^{63} \mathrm{Cu}(\mathrm{n}, \alpha)$ & 1.08 & 1.10 & 1.22 & 1.28 \\
\hline${ }^{27} \mathrm{~Np}(\mathrm{n}, \mathrm{n})$ & 1.02 & 0.76 & 1.06 & 0.81 \\
\hline${ }^{28} \mathrm{U}(\mathrm{n}, \mathrm{f})$ & 1.06 & 0.99 & 1.05 & 0.99 \\
\hline${ }^{4} \mathrm{Ti}(\mathrm{n}, \mathrm{p})$ & 1.04 & 1.06 & 1.11 & 1.12 \\
\hline
\end{tabular}

'Surveillance, Capoule at $20^{\circ}$.

${ }^{*} 0^{\circ}$ cavity location, $R=238.02 \mathrm{~cm}$. 
Table 3.5 Ratio of dosimeter reaction rates obtained with SAILOR (ENDF/B-VI Fe-O-H) to valucs obtainced with ELXSIR

\begin{tabular}{|c|c|c|c|c|}
\hline \multirow[b]{2}{*}{ Dosimeter } & \multicolumn{2}{|c|}{$\begin{array}{r}\text { ENDF/B-VI } \\
\text { dosimetry file }\end{array}$} & \multicolumn{2}{|c|}{$\begin{array}{c}\text { Original } \\
\text { dosimetry file }\end{array}$} \\
\hline & Downcomer* & Cavity*: & Downcomer * & Cavity** \\
\hline${ }^{34} \mathrm{Fe}(\mathbf{n}, \mathbf{p})$ & 1.19 & 1.43 & 1.18 & 1.42 \\
\hline${ }^{s 0} \mathrm{Ni}(\mathrm{n}, \mathrm{p})$ & 1.21 & 1.47 & 1.18 & 1.50 \\
\hline${ }^{\omega} \mathrm{Cu}(\mathrm{n}, \alpha)$ & 1.15 & 1.34 & 1.29 & 1.54 \\
\hline${ }^{207} \mathrm{~Np}(\mathrm{n}, \mathbf{n})$ & 1.11 & 1.00 & 1.15 & 1.07 \\
\hline${ }^{20} \mathrm{U}(\mathrm{n}, \mathrm{l})$ & 1.16 & 1.31 & 1.16 & 1.31 \\
\hline${ }^{*} T i(n, p)$ & 1.14 & 1.37 & 1.22 & 1.46 \\
\hline
\end{tabular}

'Surveillance Capaule at $20^{\circ}$.

**0 cavity location, $R=238.02 \mathrm{~cm}$.

Table 3.6 Impact of ENDF/B-VI Fe-O-H data on calculated cavity reaction rates for HBR

\begin{tabular}{cc}
\hline & $\frac{\text { SAlLOR (ENDF-6 Fe-O-H) }}{\text { Original SAILOR }}$ \\
\hline Dosimetcr & 1.35 \\
${ }^{34} \mathrm{Fc}(\mathrm{n}, \mathrm{p})$ & 1.34 \\
${ }^{50} \mathrm{Ni}(\mathrm{n}, \mathrm{p})$ & 1.22 \\
${ }^{60 \mathrm{Cu}(\mathrm{n}, \alpha)}$ & 1.32 \\
${ }^{27} \mathrm{~Np}(\mathrm{n}, \mathrm{n})$ & 1.32 \\
${ }^{20} \mathrm{U}(\mathrm{n}, \mathrm{n})$ & 1.30 \\
${ }^{*} \mathrm{Ti}(\mathrm{n}, \mathrm{p})$ &
\end{tabular}


Table 3.7 C/E valucs for IIIRR Cycle 9

\begin{tabular}{|c|c|c|c|c|c|c|}
\hline \multirow[b]{2}{*}{ Dosimeter } & \multicolumn{3}{|c|}{ Downcomer } & \multicolumn{3}{|c|}{ Cavity } \\
\hline & $\begin{array}{l}\text { BLXSIR } \\
\text { (Maerter) }\end{array}$ & $\begin{array}{l}\text { Original* } \\
\text { SAIIOR }\end{array}$ & $\begin{array}{l}\text { SAILOR } \\
\text { ENDF/B-VI• } \\
\text { Fe-O-II }\end{array}$ & $\begin{array}{l}\text { BLXSIR } \\
\text { (Mactker) }\end{array}$ & $\begin{array}{l}\text { Original* } \\
\text { SAIIOR }\end{array}$ & $\begin{array}{l}\text { SAIIOR } \\
\text { ENDF/B-VI* } \\
\text { Pe-O-H }\end{array}$ \\
\hline${ }^{5} \operatorname{Tr}(n, p)$ & 0.83 & 0.88 & 0.98 & 0.68 & 0.72 & 0.97 \\
\hline${ }^{s} \mathrm{Ni}(\mathrm{n}, \mathrm{p})$ & 0.87 & 0.94 & 1.05 & 0.66 & 0.72 & 0.97 \\
\hline${ }^{\infty} \mathrm{Cu}(n, \alpha)$ & 0.83 & 0.90 & 0.95 & 0.72 & 0.79 & 0.96 \\
\hline${ }^{237} N p(n, l)$ & 0.85 & 0.87 & 0.94 & 0.61 & 0.46 & 0.61 \\
\hline$U(n, n)$ & 0.80 & 0.85 & 0.93 & 0.65 & 0.65 & 0.86 \\
\hline$\| n(n, p)$ & 0.81 & 0.84 & 0.92 & 0.66 & 0.69 & 0.90 \\
\hline
\end{tabular}




\section{COMPARISON OF SAILOR, ELXSIR, AND VITAMIN-E CALCULATIONS FOR A 1-D MODEL OF HBR}

As indicated in the previous section, the availability of the new ENDF/B.VI iron cross-section data will have a major impact on transport calculations of RPV fluence. Initial results from this study indicate that the differences in the basic ENDF/B data in version VI compared with either version IV or $V$ will improve agreement with the measured HBR dosimetry. The carlicr two versions of ENDF/B (IV and V) form the basis of several cross-section libraries that have been used for years to analyze the fluence accumulated by power reactors. Some of the commonly used librarics for RPV transport calculations are SAILOR, ELXSIR, and VITAMIN-E. ${ }^{10}$

The first two were collapsed from the ENDF/B-IV based VITAMIN-C library, while VITAMIN-E, a fine-group library, is based on ENDF/B.V. As pointed out in Section 3, in the course of this study it was observed that unexpected differences occur even among some of the results obtained with the ELXSIR and the SAILOR cross-section libraries, even though the basic ENDF/B data from which these are processed are similar. These observations suggest that factors other than merely the differences between ENDF/B-VI and the earlier ENDF/B version may affect the multigroup cross-section data used by various organizations performing RPV fluence inalysis.

As part of the continuing effort to understand the causes for discrepancies between different transport calculations of RPV dosimetry, a set of 1-D calculations was performed for H.B. Robinson using SAILOR (47 groups), ELXSIR (56 groups), and VITAMIN-E (174 groups). None of the calculations use ENDF/B-VI data (i.c., SAILOR and ELXSIR are based on version IV and VITAMIN-E on version V). Hence, all three libraries are hased on similar evaluated data for the energy range of interest. The purpose of this analysis is to:

1. examine sensitivity of RPV transport calculations to iron cross-section self-shiclding and weighting spectra;

2. compare both SAILOR and ELXSIR calculations with VITAMIN-E to sec which agrees belter with a fine group calculation;

3. quantily the differences caused by the original SAILOR and ELXSIR libraries on the flux spectrum in the cavity; and

4. explain why the SAILOR results in this study differ from Macrker's carlier ELXSIR calmilations.

All transport calculations use the identical model, normalization and source spectrum (a ${ }^{23} \mathrm{U}$ Watt spectrum based on ENDF/B-V) to ensure that the observed differences reflect only the effect of the cross-section library differences.

First consider the impact of self-shielding on the VITAMIN-E calculations, as shown in Table 4.1. The first column of results used no self-shielding of the library in computing the cavity flux; the second column is the ratio indicating how much the computed values of flux above $1 \mathrm{McV}$ and above $0.1 \mathrm{McV}$, respectively, will increase if the cross sections are self-shielded correctly. The third column shows how much the integral flux values differ if the RPV iron data were self-shielded like stainless steel (SS) rather than carbon steel (CS). It can be seen that self-shielding the cross sections has a large impact on the computed cavity fluxes, even in the high-energy range above $1 \mathrm{McV}$ - the value of $\phi(>1)$ increases $25 \%$ and $\phi(>0.1)$ increases $37 \%$. Presumably it is the impact of the self-shielding on the resonances present in the iron inclastic cross section between $800 \mathrm{cV}$ and $2 \mathrm{MeV}$ that is eausing the greatest effect on the integral fluxes. At energies slightly above $2 \mathrm{MeV}$, no resolved resonance structure is shown in the iron inclastic data in ENDF/B.V. The integral fluxes are not so sensitive to resonances in the iron elastic data. The effect of self-shiclding the RPV data for a SS mixture rather than the actual CS mixture is only worth about $5 \%$ in the cavity for $\phi(>1)$ and about $10 \%$ for $\phi(>0.1)$.

In the ELXSIR library, two types of weighted iron cross sections are included. The one labeled "REM Shiclded Problem" is recommended for use in CS and the one called "Combined CTR and CRBR Processing" is for SS. Table 4.2 shows the effect of using the two differently weighted cross sections for the RPV iron data in the 
transport calculations. The SS-weighted iron produces cavity $\phi(>1)$ and $\phi(>0.1)$ that are $4 \%$ and $8 \%$ lower, respectively, than obtained with the CS.weighted data. These differences are close to those noted as a result of differences in self-shiclding for SS vs CS in VITAMIN.E. This observation suggests that most of the differences between the CS-and SS-weighted-group cross sections are probably caused by differences in self-shiclding rather than differences in the spectrum used to collapse the fine-group data. If the incorrectly weighted iron data were used in the ELXSIR calculations, a small discrepancy is introduced in the computed cavity flux.

Next, examine the impact of using SAILOR vs ELXSIR cross sections in computing the cavity flux and dosimeter activities. These conclusions are significant because SAILOR is widely used in industry for RPV fluence calculations, while ELXSIR has been used in several benchmark and sensitivity studies produced by ORNL (Macrker); hence it is important to know how to correlate the results obtained by various organizations.

Table 4.3 shows results obtained for several integral fluxes in the cavity, using the standard SAILOR and ELXSIR libraries (both of which were derived from ENDF/B-IV) and the VITAMIN.E fine group results. The VITAMIN-E values are considered to be the most accurate because of the large number of muligroups.

It can be seen that the integrated flux above $2.35 \mathrm{McV}$ is calculated only slightly lower with ELXSIR than SAILOR; therefore, the threshold dosimeters ${ }^{54} \mathrm{Fe}(n, p),{ }^{58} \mathrm{Ni}(\mathrm{n}, \mathrm{p})$ and ${ }^{63} \mathrm{Cu}(\mathrm{n}, \alpha)$ will be perhaps a litlle lower in the ELXSIR. Overall, however, the SAILOR, ELXSIR, and VITAMIN-E all agree well in the er.argy range above $2 \mathrm{MeV}$. Note however, that the integrated nux above $1 \mathrm{MeV}$ is about $8 \%$ higher in the ELXSIR calculation compared with SAILOR, and the ELXSIR value agrees well with VITAMIN.E. The SAILOR value for $\phi(>1.0)$ appears to be underestimated by about 9\% compared with VITAMIN.E. Surprisingly, the flux above $0.1 \mathrm{MeV}$ is calculated about $20 \%$ higher with ELXSIR compared with SAILOR! When compared with VITAMIN.E. SAILOR underestimates the value for $\phi(>0.1)$ by about $16 \%$. The fact that SAILOR underestimates the flux between 0.1 and $2.0 \mathrm{MeV}$ when compared with ELXSIR and VITAMIN.E will cause the lower threshold dosimeters, such as ${ }^{20} \mathrm{U}(\mathrm{n}, 1)$, and especially ${ }^{211} \mathrm{~Np}(\mathrm{n}, \mathrm{I})$, to be calculated low. Table 4.4 compares the calculated values for the neptunium fission rate. It can be seen that the ELXSIR calculation is about $20 \%$ higher than SAILOR and agrees within $6 \%$ of the VITAMIN.E value. The SAILOR value is about $12 \%$ lower than VITAMIN-E. [Note: all three calculations used the same ${ }^{21} \mathrm{~Np}(\mathrm{n}, \mathrm{n})$ cross sections.] The discrepancies between SAILOR and ELXSIR below $2 \mathrm{McV}$ are probably caused by the different group structures in the two libraries, because the impact of the different collapsing and self-shielding procedure is small. Table 4.5 shows the group structures of VITAMIN.E. Table 4.6 shows the group structures of ELXSIR and SAILOR. The ELXSIR library contains two more energy groups than SAILOR in the energy range between 0.11 and $1.0 \mathrm{MeV}$ and the bounderies are arranged somewhat differently.

Recall that the results given in Section 3 for the ${ }^{217} \mathrm{~Np}(\mathrm{n}, \mathrm{I})$ dosimeters in the HBR cavity, which were performed with the original SAILOR library, do not seem to be consistent with Macrker's earlier calculations done with ELXSIR. For example, our ${ }^{211} \mathrm{~Np}$ value in the cavity is about $24 \%$ lower than the value coniputed by Maerker. It now appears that the discrepancy is caused by differences in the group structure of the two libraries. Table 4.4 indicates the ELXSIR library produces a neptunium reaction rate about $20 \%$ higher than the corresponding SAlLOR value, due to a higher flux between 0.1 and $2 \mathrm{McV}$. These results suggest that the 47-neutron group structure in SAlL.OR may not be fine enough in the energy range 0.1 to $2.0 \mathrm{McV}$. This is evidenced by the fact that the SAILOR fluxes do not agree with the VITAMIN-E values in this energy range, while the ELXSIR does agree with the fine-group calculation. The fine-group VITAMIN-E results could be used to obtain a correction factor for the error introduced by the coarse-group structure in SAILOR. Based on the VITAMIN-E to SAILOR ratio in Table 4.4, the SAILOR ${ }^{227} \mathrm{~Np}$ values cited in Table 3.7 for the cavity dosimeter should be increased by about 14\% (l.c., 1/0.88). This correction assumes that the ENDF/B.VI SAILOR values will scale by the same factor as the original SAILOR results, and that the 2-D calculations can be scaled by a 1.D calculation. Therefore, our ocst estimate for the C/E ratios of the ${ }^{217} \mathrm{~Np}$ cavity dosimeters are 0.69 for the ENDF/B.VI SAILOR results and 0.52 for the original SAILOR results. It can be seen that our best estimate for the calculated ${ }^{207} \mathrm{~Np}$ activity in the cavity position is substantially lower than the reported measured value. Because the calculated values obtained with the ENDF/B-VI Fe-O-H SAILOR library agree well with the measured results for the other dosimeters, an experimental discrepancy is suspected for the neptunium activity. 
Table 4.1 Impact of self-shiclding on finc-group VITAMIN-E calculations in 1-D HBR moxtcl

\begin{tabular}{lccc}
\hline & $\begin{array}{c}\text { No shiclding } \\
\left(\mathrm{n} \cdot \mathrm{cm}^{-2} \cdot \mathrm{s}^{-1}\right)\end{array}$ & $\begin{array}{c}\frac{\text { Shiclding }}{\text { Nono }} \\
\left(\mathrm{n} \cdot \mathrm{cm}^{-2} \cdot \mathrm{s}^{-1}\right)\end{array}$ & $\frac{\text { SS-shicldad RPV }}{\text { CS-shiclded RPV }}$ \\
\hline Cavity $\phi(\mathrm{E}>1.0 \mathrm{MeV})$ & $2.45 \mathrm{E}-4$ & 1.25 & 0.95 \\
Cavity $\phi(\mathrm{E}>0.1 \mathrm{MeV})$ & $4.09 \mathrm{E}-3$ & 1.37 & 0.90 \\
\hline
\end{tabular}

Tablo 4.2 Impact of different cross-section weighting on ELXSIR calculations in 1-D HBR model

\begin{tabular}{lccc}
\hline & $\begin{array}{c}\text { CS-wcighted } \\
\text { RPV iron } \\
\left(\mathrm{n} \cdot \mathrm{cm}^{-2} \mathrm{~s}^{-1}\right)\end{array}$ & $\begin{array}{c}\text { SS-wcighted } \\
\text { RPV iron } \\
\left(\mathrm{n} \cdot \mathrm{cm}^{-2} \cdot \mathrm{s}^{-1}\right)\end{array}$ & $\begin{array}{c}\text { Ratio } \\
\text { (SS/CS) }\end{array}$ \\
\hline Cavity $\phi(\mathrm{E}>1.0 \mathrm{MeV})$ & $3.01 \mathrm{E}-4$ & $2.89 \mathrm{E}-4$ & 0.96 \\
Cavity $\phi(\mathrm{E}>0.1 \mathrm{MeV})$ & $5.67 \mathrm{E}-3$ & $5.28 \mathrm{E}-3$ & 0.93 \\
\hline
\end{tabular}


Table 4.3 Comparison of SAILOR and ELXSIR calculations for cavity integral nuxes in 1-D HBR model

\begin{tabular}{cccc}
\hline $\begin{array}{c}\text { Cumulative } \\
\text { nux cnergy } \\
\text { threshold (MeV) }\end{array}$ & $\begin{array}{c}\text { ELXSIR } \\
\text { SAILOR }\end{array}$ & $\frac{\text { SAILOR }}{\text { VITAMIN.E }}$ & $\frac{\text { ELXSIR }}{\text { VITAMIN.E }}$ \\
\hline 5.00 & 0.97 & 1.01 & 0.98 \\
3.00 & 0.97 & 1.04 & 1.00 \\
2.35 & 0.99 & 1.00 & 1.00 \\
1.90 & 1.01 & 0.95 & 0.96 \\
1.65 & 1.03 & 0.95 & 0.98 \\
1.35 & 1.06 & 0.92 & 0.98 \\
1.00 & 1.08 & 0.91 & 0.98 \\
0.80 & 1.11 & 0.91 & 1.01 \\
0.50 & 1.20 & 0.89 & 1.07 \\
0.10 & 1.20 & 0.84 & 1.01 \\
\hline
\end{tabular}

Table 4.4 Comparison of SAILOR and EL.XSIR calculations for cavily ${ }^{2 m} \mathrm{~Np}(n, n)$ in 1-D HBR model

\begin{tabular}{llll}
\hline EL.XSIR & $\frac{\text { SAILOR }}{\text { VITAMIN.E }}$ & $\frac{\text { ELXSIR }}{\text { VITAMIN.E }}$ \\
\hline 1.20 & 0.88 & 1.06 \\
\hline
\end{tabular}


Tuble 4.5 VITAMIN-E group structures

\begin{tabular}{|c|c|c|c|c|c|c|c|}
\hline \multicolumn{8}{|c|}{ VITAMIN-E } \\
\hline OP & $\begin{array}{c}\text { Upper E } \\
\text { (cV) }\end{array}$ & GP & $\begin{array}{c}\text { Upper E } \\
\text { (cV) }\end{array}$ & GP & $\begin{array}{c}\text { Upper 1: } \\
\text { (cV) }\end{array}$ & GP & $\begin{array}{c}\text { Upper E: } \\
\text { (cV) }\end{array}$ \\
\hline 1 & $1.9640 \mathrm{E}+07$ & 51 & $1.8268 \mathrm{E}+\left(\mathrm{X}_{6}\right.$ & 101 & $1.2 \times 17 \mathrm{E}+(105$ & 151 & $1.01 .31) \mathrm{E}+(12$ \\
\hline 2 & $1.7332 \mathrm{E}+17$ & 52 & $1.7377 \mathrm{E}+(\mathrm{K} 6$ & 112 & $1.2277 \mathrm{E}+(1) 5$ & 152 & $7.8693 \mathrm{E}+(1) 1$ \\
\hline 3 & $1.6905 \mathrm{E}+(07$ & 53 & $1.653 .3) E+\left(X_{6}\right.$ & 103 & $1.1679 \mathrm{R}+(05$ & 153 & $6.1442 E+(01$ \\
\hline 4 & $1.6487 \mathrm{E}+107$ & 54 & $1.5724 \mathrm{E}+1 \mathrm{Ko}$ & 114 & $1.11(09 \mathrm{E}+(1) 5$ & 154 & $4.78 .51 \mathrm{E}+(01$ \\
\hline 5 & $1.5683 \mathrm{E}+107$ & 55 & $1.4957 \mathrm{E}+1 \mathrm{~K}$ & 105 & $9.8(x) 37 E+(14$ & 155 & $3.7266 \mathrm{E}+(0)$ \\
\hline 6 & $1.4918 \mathrm{E}+(17$ & 56 & $1.4227 \mathrm{E}+(\mathrm{K})$ & $11(\mathbf{6}$ & $8.6517 \mathrm{E}+(1) 4$ & 156 & $2.9 \times 123 \mathrm{E}+(1) 1$ \\
\hline 7 & $1.4550 \mathrm{E}+(17$ & 57 & $1.3534 \mathrm{E}+1 \mathrm{~K} 6$ & 1177 & $8.25(1) 3 \mathrm{E}+(14$ & 157 & $2.2(x) .3 \mathrm{E}+(01$ \\
\hline 8 & $1.4191 \mathrm{E}+(07$ & 58 & $1.2874 \mathrm{E}+1 \mathrm{~K} 6$ & 1188 & $7.94(x) E+(1) 4$ & 158 & $1.76(14 \mathrm{E}+() 1$ \\
\hline 9 & 1.384() $\mathrm{E}+(1) 7$ & 59 & $1.22461 \mathrm{~L}+1 \mathrm{~K}_{6}$ & $1(x)$ & $7.199812+(14$ & 159 & $1.37101:+(01$ \\
\hline 10) & $1.349) \mathrm{E}+(17$ & (x) & $1.1648 \mathrm{E}+\left(\mathrm{K}_{6}\right.$ & 110 & $6.7379 \mathrm{E}+(14$ & (6) & 1.(K677ki+()1 \\
\hline 11 & $1.2523 \mathrm{E}+(1) 7$ & 61 & $1.1080) \mathrm{E}+(16$ & 111 & $5.6562 E+(14$ & 161 & $8.3153 \mathrm{E}+(\mathrm{X})$ \\
\hline 12 & $1.2214 \mathrm{E}+(17$ & 62 & $1 .\left(X_{1} 26 \mathrm{E}+\left(\mathrm{K}_{6}\right.\right.$ & 112 & $5.2475 E+(14$ & 162 & $6.47(x) !++(x)$ \\
\hline 13 & $1.1618 E+(1) 7$ & 63 & $9.6164 \mathrm{E}+(1) 5$ & 113 & $4.63(1) E+(1) 4$ & 163 & $5 .(435 E Z+(X)$ \\
\hline 14 & $1.1052 \mathrm{E}+(1) 7$ & 64 & $9 .(7718 E+(1) 5$ & 114 & $4.10668 \mathrm{E}+104$ & 164 & 3.9279)E $+(x)$ \\
\hline 15 & $1.051 .3 E+(17$ & 65 & $8.6294 \mathrm{E}+05$ & 115 & $3.431) 7 \mathrm{E}+1(4$ & 165 & 3.(05 $(x) E+(x)$ \\
\hline 16 & $1 .(X \times X) E+(17)$ & 66 & $8.201855 E+(05$ & 116 & $3.182812+144$ & 166 & $2.3824 \mathrm{I}+(\mathrm{X})$ \\
\hline 17 & $9.5123 \mathrm{E}+1 \times 6$ & 67 & $7.8162 \mathrm{E}+(1) 5$ & 117 & $2.8501112+(14$ & 167 & $1.85541:+(x)$ \\
\hline 18 & $9 .(1484 E+1(6)$ & 68 & $7.4274 E+(1) 5$ & 118 & $2.7(x \times) E+1) 4$ & 168 & 1.445() $\mathrm{E}+(x)$ \\
\hline 19 & $8.6(1) 71 \mathrm{E}+(\mathbf{6}$ & 69 & 7.(1651E+ (1)5 & 119 & 2.(x)58E+(1)4 & 169 & $1.125 .3 \mathrm{E}+(\mathrm{x})$ \\
\hline 21) & $8.1873 \mathrm{E}+\left(K_{5}\right.$ & 70) & $6.72(6) \mathrm{E}+1) 5$ & 120) & $2.47 \times 8 E+(1) 4$ & 17() & $8.7(64.31:-1) 1$ \\
\hline 21 & $7.78 \times(1) E+(K)$ & 71 & $6.39281:+105$ & 121 & $2.417611+(1) 4$ & 171 & $6.8256 \mathrm{~F} ;-1) 1$ \\
\hline 22 & $7.41 k_{2} 2 E+1 K_{6}$ & 72 & $6.081010 \mathrm{E}+0.5$ & 122 & $2.357918+(24$ & 172 & $5.31581:-1) 1$ \\
\hline 23 & $7 .\left(14669 \mathrm{E}+\left(\mathrm{K}_{0}\right.\right.$ & 73 & $5.78441 !+115$ & 123 & $2.1875 \mathrm{E}+(144$ & 173 & $4.1 .3(x)[:-111$ \\
\hline 24 & $6.7(1) 32 \mathrm{E}+(166$ & 74 & $5.5123 \mathrm{E}+105$ & 124 & $1.93(15 \mathrm{E}+.14$ & 174 & $1 .(X X X) \mid[-f \mid) 1$ \\
\hline 25 & $6.5924 E+1(6)$ & 75 & $5.2341) E+(1) 5$ & 125 & $1.5(3.342+(1) 4$ & & \\
\hline 26 & $6.3763 \mathrm{E}+1 \times 6$ & 76 & $4.9787 \mathrm{E}+(1) 5$ & 126 & $1.17(x) 2+14$ & & \\
\hline 27 & $6 .(16.53 \mathrm{E}+1 \mathrm{~K} 6$ & 77 & $4.5(1) 49 \mathrm{E}+(15$ & 127 & $1 .(1333.312+124$ & & \\
\hline 28 & $5.7695 \mathrm{E}+(\mathrm{K})$ & 78 & 4. $(0762 \mathrm{E}++115$ & 128 & $9.1188 \mathrm{E}+(1.3$ & & \\
\hline 29 & $5.4 \times 81 E+\left(x_{6}\right.$ & 79 & $3.8774 \mathrm{E}+(1) 5$ & 129 & $7.1(117 \mathrm{E}+(1) .3$ & & \\
\hline 30) & $5.22105 \mathrm{E}+\left(\mathrm{K}_{6}\right.$ & (x) & $3.6888 .3 \mathrm{E}+105$ & 13.) & $5.5 .3 \times \mathrm{KL}+(1) .3$ & & \\
\hline 31 & $4.9659 \mathrm{E}+16$ & 81 & $3.3373 \mathrm{E}+(0.5$ & 131 & $4.31774[2+(1) .3$ & & \\
\hline 32 & $4.7237 \mathrm{E}+166$ & 82 & 3.0197E + (1)5 & 132 & $3.7(174 \mathrm{E}+(1) 3$ & & \\
\hline 33 & $4.493 .3 \mathrm{E}+1(6$ & 83 & $2.984912+115$ & 133 & $3.3546 E+(1) 3$ & & \\
\hline 34 & $4 .\left(\mathrm{K}_{5} 57 \mathrm{E}+\left(\mathrm{K}_{6}\right.\right.$ & 84 & $2.972112+(0.5$ & 134 & $3 .(1) 354 E+(1) 3$ & & \\
\hline 35 & $3.6788 \mathrm{E}+(\mathrm{K})$ & 85 & $2.9452 \mathrm{E}+115$ & 135 & $2.7465 E+(1) .3$ & & \\
\hline 36 & $3.3287 \mathrm{E}+\left(\mathrm{X}_{6}\right.$ & 86 & $2.8725 \mathrm{E}+(1) 5$ & 136 & $2.61201 E+(1) 3$ & & \\
\hline 37 & $3.1664 E+\left(K_{1}\right)$ & 87 & $2.7 .3241 \mathrm{E}+(1) 5$ & 137 & $2.4852 E+(1) 3$ & & \\
\hline 38 & 3.(1)119E+(K6 & 88 & $2.4724 \mathrm{E}+(1) 5$ & 1.38 & $2.248712+(1) .3$ & & \\
\hline 39 & $2.8651 \mathrm{E}+\left(\mathrm{X}_{6}\right.$ & 89 & $2.3518 \mathrm{E}+(1) 5$ & 1.39 & 2.(1).347E+(1).3 & & \\
\hline 4) & $2.725 .312+(K 6$ & (Y) & $2.23711 \mathrm{E}+(0.5$ & 14) & $1.5846 E[+(1) .3$ & & \\
\hline 41 & $2.5924 \mathrm{E}+1(6$ & 91 & $2.12 \times(x) E+(0.5$ & 141 & $1.2341 E+(1) .3$ & & \\
\hline 42 & $2.4(x x) E+(16$ & 92 & $2 .(1242 \mathrm{E}+1) 5$ & 142 & $9.6112 \mathrm{E}+12$ & & \\
\hline 43 & $2.3852 \mathrm{E}+(\mathrm{K} 6$ & 93 & $1.9255 \mathrm{E}+115$ & 143 & $7.4852 \mathrm{E}+12$ & & \\
\hline 44 & $2.3653 E+(16$ & 94 & $1.831613+(1) 5$ & 144 & $5.8295 E+12$ & & \\
\hline 45 & $2.3457 E+(K 6$ & 95 & $1.7422 \mathrm{E} E+(1) 5$ & 145 & $4.54(x) E+112$ & & \\
\hline 46 & $2.3\left(K_{2}\right){ }^{2} \mathrm{E}+\left(\mathrm{K}_{6}\right.$ & $x_{0}$ & $1.65731:+105$ & 146 & $3.5357 \mathrm{E}+12$ & & \\
\hline 47 & $2.2313 E+(K 6$ & 97 & $1.57641 E+10.5$ & 147 & $2.7536 \mathrm{~B}+(1) 2$ & & \\
\hline 48 & $2.1225 \mathrm{E}+\left(K_{6}\right.$ & 98 & $1.4 \times 6 \times[3+(1) .5$ & 148 & $2.1445 E+12$ & & \\
\hline 49 & $2 .(1) 1(0) E+(K)$ & $(x)$ & $1.4264 E+(1) 5$ & 149 & $1.6712 E+12$ & & \\
\hline 5() & $1.920 .5 \mathrm{E}+16_{6}$ & $1(x)$ & $1.356(6) 1 ?+115$ & 150) & $1.3(X) 7 E+(12$ & & \\
\hline
\end{tabular}


Tuble 4.6 El.XSIR and SAII,OR group structurca

\begin{tabular}{|c|c|c|c|c|c|}
\hline \multicolumn{4}{|c|}{ ELXSIR } & \multicolumn{2}{|c|}{ SAILOR } \\
\hline OP & Upper E (cV) & OP & Upper E (cV) & GP & Upper I (cV) \\
\hline $\begin{array}{l}1 \\
2 \\
3 \\
4 \\
5 \\
6 \\
7 \\
8 \\
9 \\
10 \\
11 \\
12 \\
13 \\
14 \\
15 \\
16 \\
17 \\
18 \\
19 \\
20 \\
21 \\
22 \\
23 \\
24 \\
25 \\
26 \\
27 \\
28 \\
29 \\
30 \\
31 \\
32 \\
33 \\
34 \\
35 \\
36 \\
37 \\
38 \\
39 \\
40 \\
41 \\
42 \\
43 \\
44 \\
45 \\
46 \\
47\end{array}$ & 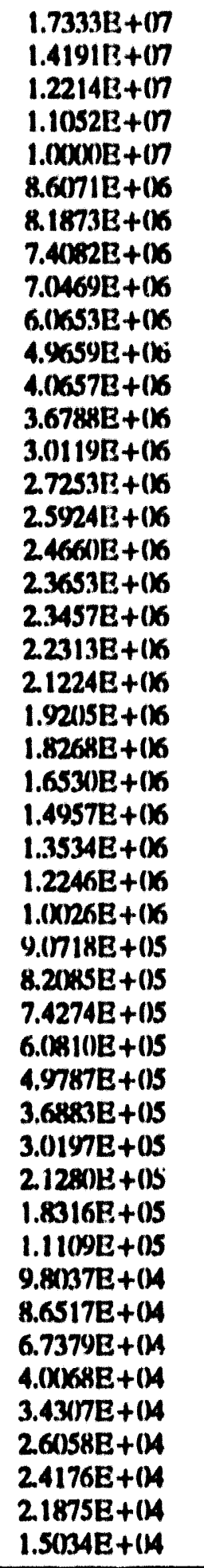 & $\begin{array}{l}48 \\
49 \\
511 \\
51 \\
52 \\
53 \\
54 \\
55 \\
56\end{array}$ & $\begin{array}{l}1.1709 \mathrm{E}+(04 \\
7.1017 \mathrm{E}+(03 \\
5.5308 \mathrm{E}+(03 \\
3.3546 \mathrm{E}+(13) \\
2.6123 \mathrm{E}+(03 \\
1.5846 \mathrm{E}+(13 \\
1.1013 \mathrm{E}+(12 \\
1.0677 \mathrm{E}+01 \\
4.1399 \mathrm{E}-101\end{array}$ & $\begin{array}{l}1 \\
2 \\
3 \\
4 \\
5 \\
6 \\
7 \\
8 \\
9 \\
11 \\
11 \\
12 \\
13 \\
14 \\
15 \\
16 \\
17 \\
18 \\
19 \\
21 \\
21 \\
22 \\
23 \\
24 \\
25 \\
26 \\
27 \\
28 \\
29 \\
31 \\
31 \\
32 \\
33 \\
34 \\
35 \\
36 \\
37 \\
38 \\
39 \\
41 \\
41 \\
42 \\
43 \\
44 \\
45 \\
46 \\
47\end{array}$ & 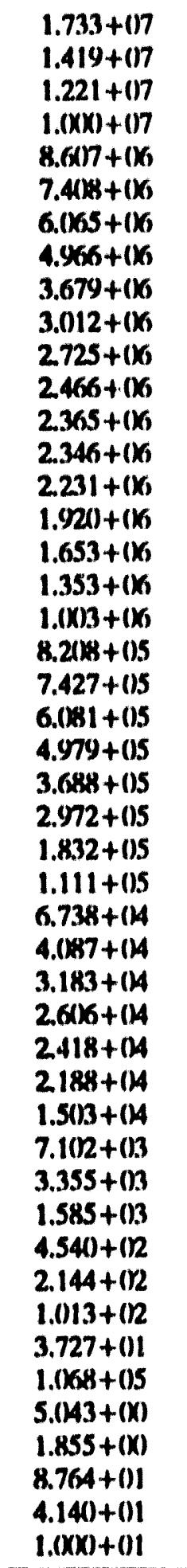 \\
\hline
\end{tabular}




\section{SUMMARY AND CONCLUSIONS}

The calculations performed with selected ENDF/B.VI data in this study have resolved most of the discrepancles between the measured and computed dosimeter activilies that were reported earlier for Cycle $9 \mathrm{H}$ of $\mathrm{H}$. Robinson. The carlicr analysis used the 56-group ELXSIR Iibrary, which is hased on ENDF/B.IV data, and obtained calculated results that were substantially lower than the measured activities in the cavity. The 47-group ENDF/B.VI calculations in this study give values that are about $2.5 \%$ higher than the corresponding dosimeter activities obtained in the earlier work. The C/E values from the ENDF/B.VI calculations agree within $10 \%$ of the measured dosimeter activities at both the downeomer and cavity measurement locations, except for the ${ }^{21} \mathrm{~Np}(\mathrm{n}, \mathrm{n})$ cavily dosimeter which is $39 \%$ 100 low. II was also discovered that the ${ }^{211} \mathrm{~Np}$ results calculated with the original 47-group SAILOR cross section differ from those obtained with ELXSIR, which is also based on ENDF/B.IV. This appears to be mostly a result of the difference in the group structure of the two libraries. The ELXSIR values agree better with fine-group calculations. It appears that the Np cavity dosimeter activity computed with the SAILOR library should be increased by about $14 \%$ in order to correct for its relatively coarse-group structure between 0.5 and $2.0 \mathrm{MeV}$. If this correction is applicd to the ENDF/B.VI calculation, then the C/E value for the neptunium becomes 0.69 , which is still about $31 \%$ low. The remaining discrepancy is believed to be caused by a problem with the experimental measurement.

It is concluded that the ENDF/B.VI cross-section data substantlally improve the accuracy of the neutron transport calculations for H. B. Robinson Cycle 9. For this particular reactor, as is the case in several other reactors studied carlier, the original SAILOR and ELXSIR data give an underestimate for the cavily dosimeter values, whlle ENDF/B.VI gives very good agreement with the experimental results. Another important conclusion of this study is that the SAILOR group structure appears to be too coarse below $2 \mathrm{McV}$, and this can cause discrepancies in low threshold responses computed with SAILOR compared to ELXSIR.

Although several addltional reactors should be analyzed prior to making a definilive assessment of using ENDF/B.VI versus curlier evaluations, the preliminary Indications suggest that RPV fluence predictions may he enhanced by establishing a "standard library" based on ENDF/B.VI data for use hy all organizations involved with pressure vessel lifetime determination. Some thought must also be given to the approprlate group structure and processing methodology for a new library in light of the apparent inconsistency with the SAIL.OR and ELXSIR librarics. 


\section{REFERENCES}

1. R. E. Maerker, L.EPRICON Analysis of Pressure Vessel Surveillance Dosimetry Inserted into H. B. Robinson.2 during Cycle 9, NUREG/CR-4439, ORNL/TM-10132, U.S Nuclcar Regulatory Commission, August 1986.

2. M. L. Williams, R. L. Childs, and M. Asgari, Analysis of H. B Rohinson PWk Vessel Fluence for Cycle 10 Utilizing Partial Length Shield Assemblies, NUREO/CR-553(), U.S. Nuclear Regulatory Commission, 1996).

3. M. L. WIIllams el al., The ELXSIR Cross-Section Library for LWR Pressure Vessel Irnadiation Studies: Part of the LEPRICON Computer Code Sysicm, EPRI NP-3654, Electric Power Research Instlfute, 1984.

4. G. L. Simmons and R. W. Roussin, RSIC Dara Library Collection-SAILOR-Coupled, Self-Shielded, 47 Neutron, 20 Gamma-Ray, P. Cross.Section Library for Light Water Reactors, DLC-76, Radiation Shiciding Information Center, Oak Ridge National Laboratory, 1985.

5. C. Y. Fu and D. M. Hetrick, Update of ENDF/B.V Mod-3 Iron: Neutron-Producing Reaction Cross Sections and Encrgy.Angle Correlations, ORNL/TM.9964 (ENDF-341), Martin Marietta Energy Systems, Inc., Oak Ridge Nall. Lub., 1986.

6. M. L. Williams et al., "Transport Calculations of Neutron Transmission Through Stecl Using ENDF/B.V, Revised ENDF/B.V, and ENDF/B.VI Iron Evaluations," Annals Nucl. En., Vol. 18, pp. 549.565, 1991.

7. R. W. Roussin et al., VITAMIN.C: The CTR Processed Multigroup Cross-Section Library for Neutronics Situdies, ORNL/RSIC-37, Union Carbide Corp., Nucl. Div., Oak Ridge Natl. Lab., 1980.

8. F. W. Stallmann, LSL.M2 Least-Squares Logarithmic Adjustment of Neutron Spectra, Radiation Shiclding Information Center, Oak Ridge National Laboralory, PSR-233 Micro, 1985.

9. E. P. Lippincolt et al., Evaluation of Survcillance Capsule and Reactor Cavity Dosimetry from H. B. Robinson Unit 2, Cycle 9, NUREG/CR.4576, WCAP.11104, Westinghouse Corp., February 1987.

11). C. R. Weishin el al., VITAMIN-E: An ENDF/B.V Mulligroup Cross-Section Library for LMFBR Core and Shield, LWR Shicld, Dosimery and Fusion Blanke' Technology, ORNL-5505 (ENDF-274), Union Carbide Corp., Nucl. Div., Oak Ridge Nall. Lah., 1979. 
NUREO/CR-6071

Version 1

ORNL/TM-12406

Dist. Category R5

\section{INTERNAL DISTRIBUTION}

$\begin{aligned} \text { 1. } & \text { B. R. Appleton } \\ 2 . & \text { W. R. Conwin } \\ 3 . & \text { S. K. Iskander } \\ 4.23 . & \text { F. B. K. Kam } \\ 24 . & \text { R. K. Nanstad } \\ 25 . & \text { W. E. Pennell } \\ 26 . & \text { C. E. Pugh } \\ 27 . & \text { I. Remec } \\ 28 . & \text { C. H. Shappert } \\ 29.32 . & \text { F. W. Stallmann }\end{aligned}$

$\begin{aligned} 33.36 . & \text { J. A. Wang } \\ 37 . & \text { R. M. Westfall } \\ 38 . & \text { G. E. Whitesides } \\ 39 . & \text { Ceniral Rescarch Library } \\ 40 . & \text { ORNL Y.12 Rescarch Library } \\ & \text { Document Reference Section } \\ 41.42 . & \text { Laboratory Records Departyment } \\ 43 . & \text { Laboratory Records, ORNL (RC) } \\ 44 . & \text { ORNL Patent Offlce }\end{aligned}$

\section{EXTERNAL DISTRIBUTION}

45. Ait Abderrahim, SCK/CEN Fucl Rescarch Unit, Boeretang, 200B-2400 MOL, Belgium

46. S. L. Anderson, Radiation and Environmental Systems, Westinghouse Electric Corporation, Nuclear Energy Systems, Monrocville Nuclear Center, P.O. Box 355, Pittsburgh, PA 15230

47. M. Asgari, Loulsiana State University, Nuclear Science Center, Baton Rouge, LA 70803

48. J. F. Carew, Bldg. 130 Department of Nuclear Engineering, Brookhaven National Laboratory, Upton Long Island, New York 11973

49. G. P. Cavanaugh, Combustion Enginecring, Inc., Dept. 9492-425, 1000 Prospect Hill Rd., Windsor, CT 06095

50. J. Helm, Dept. of Applied Physics \& Nuclear Enginecring, 202 Mudd, Columbia University, Now York, New York $10(1) 27$

51. A. Hiser, U.S. Nuclear Regulatory Commission, Division of Engincering, Mail Stop NL S 217 C, Washington, DC 20555

52. W. C. Hopkins, Bechtel Corporation, 98011 Washingtonian Blvd., Gaithersburg, MD 20878.5356

53. K. D. llieva, Academy of Science, Institute of Nuclear Rescarch and Nuclear Energy, Boul, Tzarigradsko, Shausce 72

54. E. P. Lippincott, Westinghouse Electric Corporation, M/S E4-33, Westinghouse Energy Center, P.O. Box 355, Piltsburgh, PA 15230-0355

55. L. Lois, U.S. Nuclear Regulatory Commission, Phillips Bullding, MS P.924, Washington, DC 20555 
56. M. Mayficld, U.S. Nuclear Regulatory Commission, Division of Engineering, Mail Stop NL S 217 C, Washinglon, DC 20555

57. E. D. McGarry, National Institute of Standards and Technology, Bldg. 235-A155, Gaithersburg, MD 20899

58. B. Osmera, Nuclear Rescarch Institute, Narcony Trida 3, 25068 Rez, Czechoslovakia

59. J. Rataj, 25068 Rez, Czechoslovakia

60. E. Sajo, Louisiana State University, Nuclear Science Center, Baton Rouge, LA 70803

61. C. Z. Serpan, Jr., U.S. Nuclear Regulatory Commission, Division of Engineering, Mail Stop NL S 217C, Washington, DC 20555

62. Al Taboada, U.S. Nuclear Regulatory Commission, Division of Engineering, Mail Stop NL. $S$ $217 \mathrm{C}$, Washington, DC 20555

63. M. L. Williams, Louisiana State University, Nuclear Science Center, Baton Rouge, LA 70803

64. S. Zaritsky, Kurchatov Institute, Kurchatov Square, 123182 Moscow, Russia

65. E. Zsolnay, Technical University of Budapest, BME H-1521 Budapest, Muegytem RKP9, Hungary

66. Office of the Deputy Assistant Manger for Encrgy, Research and Development, Department of Energy Oak. Ridge Operations (DOE-ORO), P.O. Box 2008, Oak Ridge, TN 37831-6269

67-68. Office of Scientific and Technical Information, P.O. Box 62, Oak Ridge, TN 37831 

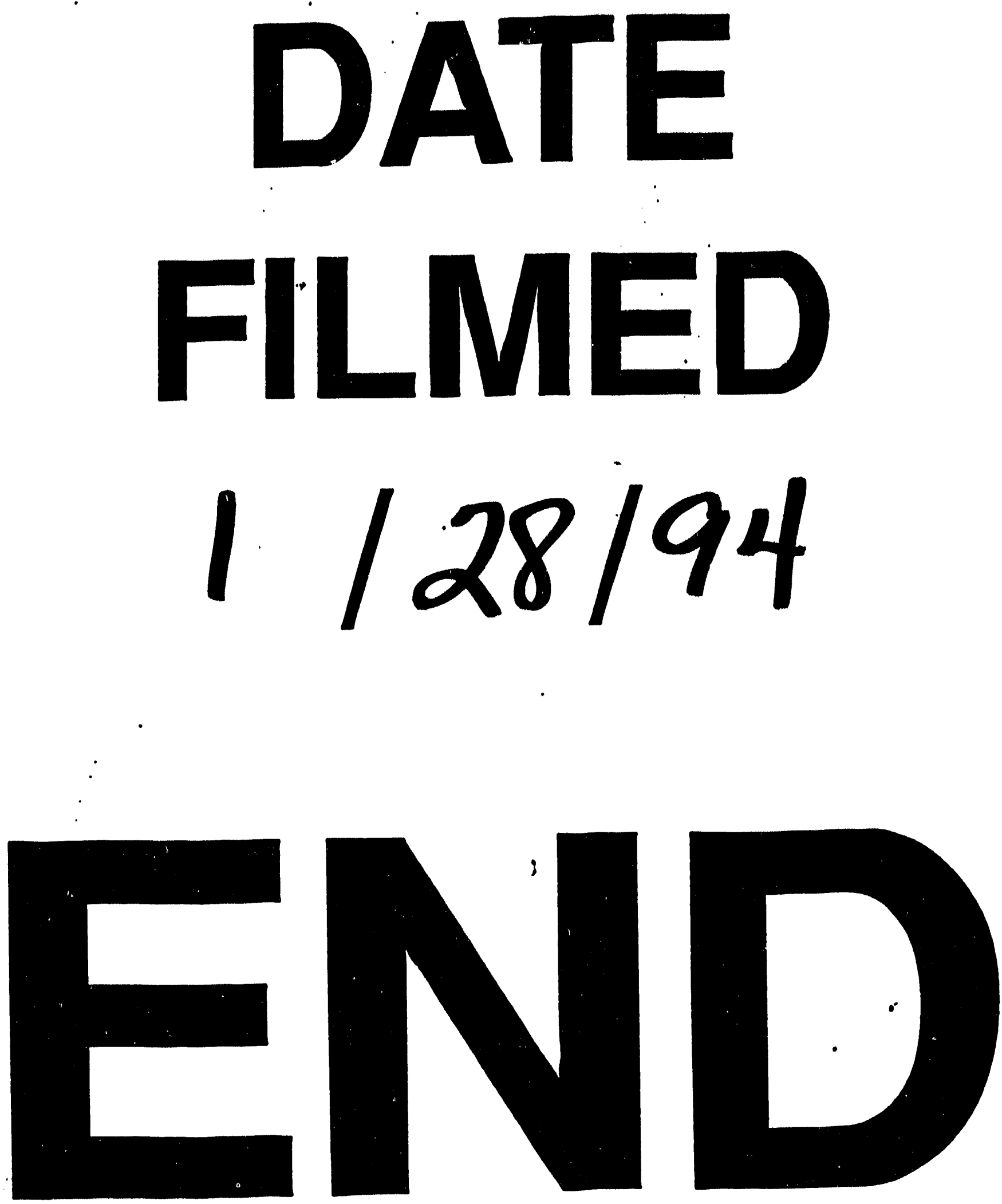
